\title{
ONE-DIMENSIONAL BAD NOETHERIAN DOMAINS
}

\author{
BRUCE OLBERDING
}

\begin{abstract}
Local Noetherian domains arising as local rings of points of varieties or in the context of algebraic number theory are analytically unramified, meaning their completion has no nontrivial nilpotent elements. However, looking elsewhere, many sources of analytically ramified local Noetherian domains have been exhibited over the last seventy-five years. We give a unified approach to a number of such examples by describing classes of DVRs which occur as the normalization of an analytically ramified local Noetherian domain, as well as some that do not occur as such a normalization. We parameterize these examples, or at least large classes of them, using the module of Kähler differentials of a relevant field extension.
\end{abstract}

\section{INTRODUCTION}

As early as 1935, examples were known of Noetherian rings differing in fundamental ways from the Noetherian rings arising in algebraic geometry or algebraic number theory. In that year, fourteen years after Noether introduced the ascending chain condition into ideal theory, Akizuki and Schmidt each gave examples of one-dimensional local Noetherian domains failing to have finite normalization, where a ring $A$ has finite normalization if its integral closure in its total ring of quotients is a finitely generated $A$-module [1,30. Hence by a theorem of Krull, since the rings of Akizuki and Schmidt are one-dimensional and do not have finite normalization, they have nontrivial nilpotents in the completion; that is, the rings are analytically ramified [13. Over the last seventy-five years, other constructions of one-dimensional analytically ramified local Noetherian domains have been developed; see for example [1, 2, 4, 6, 8, 10, 16, 21, 25, 27, 30, 1] The most well-known example perhaps is in Example 3 of an appendix of Nagata's Local Rings titled, "Examples of bad Noetherian rings". In this article we seek to unify a number of these examples by considering the question of which rank one discrete valuation rings (DVRs) can occur as the normalization of an analytically ramified local Noetherian domain.

In considering these examples, certain themes predominate. For example, the constructions of Akizuki, Schmidt and Nagata all occur in an immediate extension of DVRs. More precisely, the examples of Akizuki, Schmidt and Nagata are of an analytically ramified local Noetherian $\operatorname{ring} R$ such that $U \subseteq R \subseteq V$, where $U$ and $V$ are DVRs with $V=U+\mathfrak{M}_{U} V$ (here, as throughout the paper, $\mathfrak{M}_{U}$ denotes 2012 .

Received by the editors April 18, 2011 and, in revised form, February 6, 2012 and July 15,

2010 Mathematics Subject Classification. Primary 13E05, 13B35, 13B22; Secondary 13 F40.

${ }^{1}$ Although of a different spirit, Lech's construction in [15] of local Noetherian domains having prescribed completion can also be used to produce analytically ramified local Noetherian domains. Where the references cited above differ is in an emphasis on prescribed normalization rather than completion. For a survey that touches on each of the cited references, see [26]. 
the maximal ideal of the valuation ring $U$ ), and $V$ is the integral closure of $R$ in its quotient field (i.e., $V$ is the normalization of $R$ ). The choice of immediate extension $U \subseteq V$ is different for each of these authors; for example, Schmidt and Nagata require characteristic $p$, whereas Akizuki does not. On the other hand, the examples of Schmidt and Nagata are simple integral extensions of the DVR $U$, while Akizuki's example is not even finite over $U$.

One of the main goals of this article is to formalize what it is about the choices of $U$ and $V$ that permits analytically ramified local Noetherian rings to be situated between them. This proves to depend only on the quotient fields $Q$ and $F$ of $U$ and $V$, respectively: In Corollary 4.2, we show there exists an analytically ramified local Noetherian domain containing $U$ and having normalization $V$ if and only if either (a) $F$ has characteristic 0 and $F / Q$ is not algebraic, or (b) $F$ has characteristic $p>0$ and $F \neq Q\left[F^{p}\right]$. Statements (a) and (b) can be compressed into the single assertion that the module of Kähler differentials $\Omega_{F / Q}$ for the field extension $F / Q$ does not vanish. In fact, we show in Theorem 4.1 that each proper full $V$-submodule $K$ of $\Omega_{F / Q}$ yields a different analytically ramified local Noetherian domain $R$ with normalization $V$, the ring $R$ consisting of "antiderivatives" for $K$ in $V$; that is, $R=V \cap d_{F / Q}^{-1}(K)$, where $d_{F / Q}: F \rightarrow \Omega_{F / Q}$ is the exterior differential of the field extension $F / Q$.

While not every one-dimensional analytically ramified local Noetherian domain containing $U$ and having normalization $V$ is captured in this way, it is possible using Theorem 4.1 to describe precisely the rings obtained from the exterior differential. They are the analytically ramified local Noetherian domains $R$ such that every ideal of $R$ has a principal reduction of reduction number at most 1; equivalently, $\widehat{R}$ has a nonzero prime ideal $P$ such that $P^{2}=0$ and $\widehat{R} / P$ is a DVR. Following Lipman [17] and Sally and Vasconcelos [29], such rings are termed stable. Stable rings are discussed in Section 2.

Although a one-dimensional analytically ramified local Noetherian domain $R$ need not be stable, such a ring must have an analytically ramified stable ring between it and its quotient field (see the discussion that precedes Corollary 4.2). This observation, along with the material in Sections 4 and 5, is applied in Sections 6 and 7 to the problem of determining which DVRs occur as the normalization of an analytically ramified local Noetherian domain. For example, we show that any uncountable DVR of equicharacteristic 0 (e.g., a complete DVR of equicharacteristic 0 ) is for each $d>0$ the normalization of an analytically ramified local Noetherian stable domain of embedding dimension $d$ (Corollary 7.2). Similar statements hold when $V=\widehat{\mathbb{Z}}_{(p)}$ or $V=k[[X]]$ for a field $k$ of positive characteristic $p$ such that $\left[k: k^{p}\right]$ is uncountable (Theorem $7.6(2)$ ). By contrast, if $k$ is a perfect field of positive characteristic, then $k[[X]]$ is not the normalization of an analytically ramified local Noetherian domain (Theorem[7.6(1)). In general, a DVR $V$ of positive characteristic $p$ with quotient field $F$ is the normalization of an analytically ramified local Noetherian domain if and only if there is a subfield $Q$ of $F$ such that $F=Q+V$ and $F \neq Q\left[F^{p}\right]$ (Theorem 6.5). All of these results concern raw existence of an analytically ramified local Noetherian domain with prescribed normalization, but in the case of an algebraic function field $F / k$ we are able to assert existence within the category of $k$-algebras: In function fields the valuation rings with finitely generated residue field that are the normalization of an analytically ramified local Noetherian domain in the function field are precisely the nondivisorial DVRs (Theorem 7.3). 
Throughout the article we focus on the unibranched case: local Noetherian domains having normalization a DVR. In general, basic ideas allow one to reduce things to consideration of one-dimensional analytically ramified local Noetherian domains $R$ having normalization a DVR; see for example [2, Section 1] or the proof of Theorem 5.10 in $[25]$.

All rings considered are commutative and have an identity. In addition, we use the following standard notation and terminology. The normalization $\bar{R}$ of a ring $R$ is the integral closure of $R$ in its total ring of quotients. The ring $R$ has finite normalizaton if $\bar{R}$ is a finitely generated $R$-module. When $R$ is a quasilocal ring with maximal ideal $M$, then we denote by $\widehat{R}$ the completion of $R$ in the $M$-adic topology. When $V$ is a valuation domain, we denote its maximal ideal by $\mathfrak{M}_{V}$. An extension of valuation domains $U \subseteq V$ is immediate if $U$ and $V$ share the same value group and residue field. If also $U$ and $V$ are DVRs, then $U \subseteq V$ is immediate if and only if $\mathfrak{M}_{U} \subseteq \mathfrak{M}_{V}$ and $V=U+\mathfrak{M}_{U} V$; if and only if $V / U$ is a torsion-free divisible $U$-module.

\section{Preliminaries on Derivations and Stable Rings}

In this section we review some technical properties of derivations that are needed later, and we briefly discuss the class of one-dimensional stable rings. Let $S$ be a ring, $R$ a subring of $S$, and $L$ an $S$-module. Given a subset $A$ of $S$, an $A$-linear derivation $D: S \rightarrow L$ is an $A$-linear mapping such that $D(s t)=s D(t)+t D(s)$ for all $s, t \in S$. The main properties of derivations we need are collected in (2.1) and $(2.2)$.

(2.1) The module $\Omega_{S / A}$ of Kähler differentials. Let $A \subseteq S$ be an extension of rings. There exists an $S$-module $\Omega_{S / A}$, along with an $A$-linear derivation $d_{S / A}$ : $S \rightarrow \Omega_{S / A}$, such that for every derivation $D: S \rightarrow L$, there is a unique $S$-module homomorphism $\alpha: \Omega_{S / A} \rightarrow L$ with $D=\alpha \circ d_{S / A}$; see for example, [14, Theorem 1.19, p. 12]. The actual construction of $\Omega_{S / A}$ is not needed here, but we do use the fact that the image of $d_{S / A}$ in $\Omega_{S / A}$ generates $\Omega_{S / A}$ as an $S$-module 14, Remark 1.21, p. 13]. The $S$-module $\Omega_{S / A}$ is the module of Kähler differentials of the ring extension $A \subseteq S$, and the derivation $d_{S / A}: S \rightarrow \Omega_{S / A}$ is the exterior differential of $A \subseteq S$.

(2.2) Kähler differentials of field extensions. When $F / Q$ is an extension of fields, then $\Omega_{F / Q}$ is an $F$-vector space, and hence its structure is determined entirely by its dimension, $\operatorname{dim}_{F} \Omega_{F / Q}$. If $F / Q$ is a separably generated extension, then $\operatorname{dim}_{F} \Omega_{F / Q}=\operatorname{tr} . \operatorname{deg}_{Q} F$ [14, Corollary 5.3, p. 74]. If $Q$ has characteristic $p \neq 0$, then $\operatorname{dim}_{F} \Omega_{F / Q}$ is the cardinality of a $p$-basis of $F$ over $Q$ [14, Proposition 5.6, p. 76]. Of particular interest is the case where $\Omega_{F / Q}=0$. Recall that for a field $F$ of characteristic $p \neq 0, F^{p}$ denotes the subfield consisting of the $p$-powers of elements in $F$, and if $Q$ is a subfield of $F$, then $Q\left[F^{p}\right]$ is the subfield of $F$ generated by $Q$ and $F^{p}$. Using the above ideas, along with the existence of a $p$-basis, the case where $\Omega_{F / Q}=0$ can be discerned: When $F$ has characteristic $0, \Omega_{F / Q}=0$ if and only if $F$ is algebraic over $Q$; otherwise, when $F$ has prime characteristic $p$, $\Omega_{F / Q}=0$ if and only if $F=Q\left[F^{p}\right]$ [14, Proposition 5.7].

Following Lipman [17] and Sally and Vasconcelos [29], an ideal $I$ of a ring $R$ is stable if $I$ is projective over its ring of endomorphisms. If every ideal of $R$ containing a nonzerodivisor is stable, then $R$ is a stable ring. When $R$ is a quasilocal domain 
(the main case which we consider in the article), stable ideals $I$ are characterized by the property that there exists $i \in I$ such that $I^{2}=i I$ [22, Lemma 3.1]. Thus a quasilocal domain $R$ is stable if and only if every ideal has a principal reduction of reduction number $\leq 1$.

(2.3) Noetherian stable rings. Since every ideal of a quasilocal stable domain has a principal reduction, and hence the radical of every proper ideal is the radical of a principal ideal, it follows that a local Noetherian stable ring has Krull dimension 1. A local Noetherian ring $R$ with finite normalization is a stable ring if and only if $R$ is a 2-generator ring, meaning that every ideal of $R$ can be generated by 2 elements [29, Theorem 2.4]. However, if $R$ does not have finite normalization, then $R$ need not be a 2-generator ring. Examples of such rings were given by Sally and Vasconcelos 29] and Heinzer, Lantz and Shah [9] using a construction of Ferrand and Raynaud over a specific field of characteristic 2. We will exhibit large classes of stable rings without finite normalization in this article; other sources of examples can be found in 25 .

(2.4) Bad stable rings. Among stable rings, our main focus is on the class of one-dimensional quasilocal stable domains $R$ without finite normalization $\bar{R}$. We refer to such rings as bad stable rings, where "bad" is in the sense of Nagata's appendix, "Examples of bad Noetherian rings", in [21]. There is a helpful extrinsic characterization of bad stable rings in terms of the extension $R \subseteq \bar{R}$ : A quasilocal domain $R$ is a bad stable ring if and only if $\bar{R}$ is a DVR, $\bar{R} / R$ is a divisible $R$ module, and every $R$-submodule of $\bar{R}$ containing $R$ is a ring; see [25. Proposition 2.1]. In the next section, we express the last condition by saying $R \subseteq \bar{R}$ is a quadratic extension. Using this characterization, it is shown in [25, Theorem 4.2] that a quasilocal domain $R$ with quotient field $F$ is a bad stable ring if and only if $\bar{R}$ is a DVR and $\bar{R} / R \cong \bigoplus_{i \in I} F / \bar{R}$ for some index set $I$. This in turn yields a characterization of bad Noetherian stable rings of embedding dimension $n$ as those for which the index set $I$ can be chosen to have $n-1$ elements [25. Corollary 4.3]. In particular, $R$ is a bad 2-generator ring (that is, a local 2-generator ring without finite normalization) if and only if $\bar{R}$ is a DVR and $\bar{R} / R \cong F / \bar{R}$.

(2.5) The completion of a bad stable ring. Corollary 7.7 shows that a bad stable ring need not be Noetherian. This subtlety forces us to frame the analytic characterization of bad stable rings in terms of the completion of $R$ in the ideal topology, $\widetilde{R}=\lim R / r R$, where $r$ ranges over all nonzero elements of $R$. When $R$ is onedimensional Noetherian, or stable, it is easy to see that $\widetilde{R}$ is isomorphic to the usual completion $\widehat{R}$ of $R$ in its $M$-adic topology. In any case, a quasilocal domain $R$ is a bad stable ring if and only if there is a nonzero prime ideal $P$ of $\widetilde{R}$ with $P^{2}=0$ and $\widetilde{R} / P$ is a DVR [25, Theorem 3.4]. Thus, among Noetherian rings, the bad stable domains are characterized by the property that there exists a nonzero prime ideal $P$ of the $M$-adic completion $\widehat{R}$ of $R$ such that $P^{2}=0$ and $\widehat{R} / P$ is a DVR.

\section{AnAlytic isomorphisms}

The purpose of this section is prove some general results that in the next section will specialize to some of our main technical tools for dealing with rings in an immediate extensions of DVRs. The justifications for the results in this section 
involve some technical but elementary arguments which we give in a very general setting. Our motivation for working at this level of abstraction is twofold. First, it comes at no extra expense of proof, since our particular setting involving DVRs does not seem to simplify or shorten the arguments. Second, in [24], we apply the lemmas as stated here to a more general context in which this level of abstraction is needed.

Let $\alpha: K \rightarrow L$ be a homomorphism of $A$-modules, and let $C$ be a multiplicatively closed subset of $A$ consisting of nonzerodivisors of $A$. Following Weibel in 31, we say that $\alpha$ is an analytic isomorphism along $C$ if for each $c \in C$, the induced mapping $\alpha_{c}: K / c K \rightarrow L / c L: a+c K \mapsto \alpha(a)+c L$ is an isomorphism. It follows that $\alpha$ is analytic along $C$ if and only if $\alpha(K) \cap c L=c \alpha(K)$ and $L=\alpha(K)+c L$ for all $c \in C$. We express the former condition by saying that $L / \alpha(K)$ is a $C$-torsionfree $A$-module, and the latter by saying that $L / \alpha(K)$ is a $C$-divisible $A$-module. Similarly, an $A$-module $T$ is $C$-torsion provided that for each $t \in T$, there exists $c \in C$ with $c t=0$.

An extension $R \subseteq S$ is quadratic if every $R$-submodule of $S$ containing $R$ is a ring; equivalently, st $\in s R+t R+R$ for all $s, t \in S$. In light of the fact that the inclusion of a bad stable ring in its normalization is a quadratic extension (2.4), the following lemma, taken from [25. Lemma 3.2], is relevant in a number of results that follow.

Lemma 3.1. Let $R \subseteq S$ be an extension of rings, and let $C$ be a multiplicatively closed subset of $R$ consisting of nonzerodivisors of $S$. If the $R$-module $S / R$ is $C$ torsion and $C$-divisible, then the following statements are equivalent:

(1) $R \subseteq S$ is a quadratic extension of rings.

(2) There exist an $S$-module $T$ and a derivation $D: S \rightarrow T$ with $R=\operatorname{Ker} D$.

(3) For all $c \in C,(R \cap c S)^{2} \subseteq c R$.

(4) $S / R$ admits an $S$-module structure extending the $R$-module structure.

The next lemma, which establishes the existence of a derivation associated to certain subrings between $A$ and $S$, is the key technical result needed to frame the correspondence in Proposition 3.3 and for immediate extensions of DVRs in Theorem 4.1. The proof uses the notion of Nagata idealization (or trivialization) of a module. Let $B$ be a ring and $L$ be a $B$-module. Then the idealization $B \star L$ of $L$ is defined as a $B$-module to be $B \oplus L$, and whose ring multiplication is given by $\left(b_{1}, \ell_{1}\right) \cdot\left(b_{2}, \ell_{2}\right)=\left(b_{1} b_{2}, b_{1} \ell_{2}+b_{2} \ell_{1}\right)$ for all $b_{1}, b_{2} \in B$ and $\ell_{1}, \ell_{2} \in L$. Thus the ring $B \star L$ has a square zero ideal corresponding to $L$. In analogy with internal direct sums, we can consider decompositions of rings into idealizations. Thus if $A$ is a subring of $B$ and $I$ is an ideal of $B$, then we write $B=A \star I$ when $B=A+I$, $0=A \cap I$, and $I^{2}=0$. It is straightforward to see that the existence of such a decomposition is equivalent to the presence of a derivation: $B=A \star I$ if and only if there exists a derivation $D: B \rightarrow I$ such that $A=\operatorname{Ker} D$ and $D(i)=i$ for all $i \in I$; cf. [14, Proposition 1.17, p. 11].

Lemma 3.2. Let $A \subseteq R \subseteq S$ be an extension of rings such that $R \subseteq S$ is a quadratic extension, let $C$ be a multiplicatively closed subset of $A$ consisting of nonzerodivisors of $S$, and suppose that the inclusion mapping $A \rightarrow S$ is an analytic isomorphism along $C$. If $S / R$ is $C$-torsion, then there exists an A-linear derivation 
$D: S \rightarrow L$, where $L$ is a C-torsion-free $S$-module, such that $D(S)=c D(S)$ for each $c \in C$ and $R=D^{-1}(K)$ for some $S$-submodule $K$ of $L$ such that $L / K$ is C-torsion.

Proof. Define $\widetilde{R}=\lim _{\longleftarrow} R / c R$ and $\widetilde{S}=\lim _{\longleftarrow} S / c S$, where $c$ ranges over the elements of $C$. We denote the elements of $\widetilde{R}$ by $\left\langle r_{c}+c R\right\rangle$, where each $r_{c} \in R$ and for $c, d \in C$, $r_{c}-r_{c d} \in c R$. The elements of $\widetilde{S}$ are represented similarly as $\left\langle s_{c}+c S\right\rangle$. Let $I$ be the kernel of the canonical ring homomorphism $\phi: \widetilde{R} \rightarrow \widetilde{S}$; i.e.,

$$
I=\left\{\left\langle r_{c}+c R\right\rangle \in \widetilde{R}: r_{c} \in c S \text { for all } c \in C\right\} .
$$

Define a subring $A^{\prime}$ of $\widetilde{R}$ by

$$
A^{\prime}=\left\{x \in \widetilde{R}: x=\left\langle a_{c}+c R\right\rangle, a_{c} \in A \text { for all } c \in C\right\} .
$$

We first prove the following claim.

There is a derivation $D_{0}: \widetilde{R} \rightarrow I$ such that $A^{\prime}=\operatorname{Ker} D_{0}$ and $D_{0}(x)=x$ for all $x \in I$.

By the observation preceding the lemma, it is enough to show that $\widetilde{R}=A^{\prime} \star I$. Let $\left\langle r_{c}+c R\right\rangle \in \widetilde{R}$. Then since $S / A$ is $C$-divisible, for each $c \in C$ there exist $a_{c} \in A$ and $s_{c} \in S$ such that $r_{c}=a_{c}+c s_{c}$. We claim that $\left\langle a_{c}+c R\right\rangle \in A^{\prime}$ and $\left\langle c s_{c}+c R\right\rangle \in I$. Since each $a_{c} \in A$, the first assertion amounts to showing that $\left\langle a_{c}+c R\right\rangle \in \widetilde{R}$. Let $c, d \in C$. Then since $r_{c}-r_{c d} \in c R$, we use the fact that $S / A$ is $C$-torsion-free to obtain

$$
a_{c}-a_{c d}=r_{c}-r_{c d}+c d s_{c d}-c s_{c} \in c S \cap A \subseteq c R,
$$

and hence $\left\langle a_{c}+c R\right\rangle \in \widetilde{R}$. This also shows that $c d s_{c d}-c s_{c} \in c R$, and hence that $\left\langle c s_{c}+c R\right\rangle \in \widetilde{R}$. Therefore, $\left\langle c s_{c}+c R\right\rangle \in I$, and we have proved that $\widetilde{R}=A^{\prime}+I$. Again using the fact that $S / A$ is $C$-torsion-free, we conclude that $\widetilde{R}=A^{\prime} \oplus I$. For if $\left\langle a_{c}+c R\right\rangle \in A^{\prime} \cap I$, then for each $c \in C, a_{c} \in c S \cap A \subseteq c R$, so that $\left\langle a_{c}+c R\right\rangle=0$.

Thus to see that $\widetilde{R}=A^{\prime} \star I$, it remains to show that $I^{2}=0$. Let $\left\langle c s_{c}+c R\right\rangle,\left\langle c s_{c}^{\prime}+\right.$ $c R\rangle \in I$, where $s_{c}, s_{c}^{\prime} \in S$ with $c s_{c}, c s_{c}^{\prime} \in R$. By assumption, $R \subseteq S$ is quadratic and the $R$-module $S / R$ is $C$-torsion. Also, since $S / A$ is $C$-divisible, so is $S / R$. Therefore, Lemma 3.1 is applicable, so that by (3) of this lemma, for each $c \in C$ we have $\left(c s_{c}\right)\left(c s_{c}^{\prime}\right) \in(c S \cap R)^{2} \subseteq c R$, and hence $\left\langle c s_{c}+c R\right\rangle\left\langle c s_{c}^{\prime}+c R\right\rangle=0$. Therefore, $I^{2}=0$, and we conclude that $\widetilde{R}=A^{\prime} \star I$. This proves the claim that there is a derivation $D_{0}: \widetilde{R} \rightarrow I$ such that $A^{\prime}=\operatorname{Ker} D_{0}$ and $D_{0}(x)=x$ for all $x \in I$.

We now prove the lemma. With $D_{0}$ and $I$ as above, define an $A$-linear derivation $D: R \rightarrow I$ by $D(r)=D_{0}(\langle r+c R\rangle)$ for all $r \in R$. Since $\widetilde{R}$ is $C$-torsion-free and $I \subseteq \widetilde{R}$, then $I$ is also $C$-torsion-free. We extend $D$ to a $C$-linear derivation $D: R_{C} \rightarrow I_{C}$ by defining for all $r \in R$ and $c \in C$ :

$$
D\left(\frac{r}{c}\right)=\frac{1}{c} D(r)
$$

We view $I$ as an $S$-module in the following way. For each $s \in S$ and $\left\langle r_{c}+c R\right\rangle \in I$, define $s \cdot\left\langle r_{c}+c R\right\rangle=\left\langle a_{c} r_{c}+c R\right\rangle$, where for each $c \in C, s=a_{c}+c \sigma_{c}$, with $a_{c} \in A$ and $\sigma_{c} \in S$. (Here we are using the fact that $S / A$ is $C$-divisible.) The operation . is well defined, since if for some $c \in C, s=b+c \tau$, with $b \in A$ and $\tau \in S$, then by Lemma 3.1 $a_{c} r_{c}-b r_{c}=\left(\tau-\sigma_{c}\right) c r_{c} \in(c S \cap R)^{2} \subseteq c R$. (Here we are using the fact that $\left\langle r_{c}+c R\right\rangle \in I$ implies $r_{c} \in c S \cap R$.) It is now easily checked that - defines an $S$-module structure on $I$ that extends the $R$-module structure on $I$. 
We define $K$ to be the $S$-submodule of $I$ generated by $D(R)$. Since $S \subseteq R_{C}$, we may apply $D$ to $S$ and define $L$ to be the $S$-submodule of $K_{C}$ generated by $D(S)$. Observe that $L / K$ is $C$-torsion since $L \subseteq K_{C}$. Moreover, $\left.D\right|_{S}: S \rightarrow L$ is a derivation that extends the derivation $\left.D\right|_{R}: R \rightarrow K$.

To see that $R=S \cap D^{-1}(K)$, suppose that $s \in S$ such that $D(s) \in K$. Then since $S / R$ is $C$-torsion, there exists $d \in C$ such that $d s \in R$, and, since $D$ is $C$ linear, $D(d s)=d D(s) \in d K$. Thus since $K \subseteq \widetilde{R}$, there exists $\left\langle r_{c}+c R\right\rangle \in \widetilde{R}$ with $D(d s)=\left\langle d r_{c}+c R\right\rangle$. Now as we have shown, $\widetilde{R}=\operatorname{Ker} D_{0}+I$, so

$$
\langle d s+c R\rangle=\left\langle a_{c}+c R\right\rangle+\left\langle c \sigma_{c}+c R\right\rangle,
$$

where $\left\langle a_{c}+c R\right\rangle \in \operatorname{Ker} D_{0}$ and $\left\langle c \sigma_{c}+c R\right\rangle \in I$ (and each $\sigma_{c}$ is in $S$ with $c \sigma_{c} \in R$ ). Thus since $D_{0}(x)=x$ for all $x \in I$,

$$
D(d s)=D_{0}(\langle d s+c R\rangle)=\left\langle c \sigma_{c}+c R\right\rangle,
$$

and pairing this with our previous representation of $D(d s)$, we conclude that $\left\langle c \sigma_{c}+\right.$ $c R\rangle=\left\langle d r_{c}+c R\right\rangle$. In particular, $d \sigma_{d}-d r_{d} \in d R$, and since $d$ is a nonzerodivisor in $S, \sigma_{d}-r_{d} \in R$. Consequently, $\sigma_{d} \in R$. Moreover, from $\langle d s+c R\rangle=\left\langle a_{c}+c R\right\rangle+$ $\left\langle c \sigma_{c}+c R\right\rangle$, we see that there exists $\rho \in R$ such that $d s-d \sigma_{d}-d \rho \in A \cap d S \subseteq d R$ (here we are using the fact that $S / A$ is $C$-torsion-free). Since $d$ is a nonzerodivisor in $S$, this implies that $s-\sigma_{d}-\rho \in R$. Since $\rho \in R$ and we have shown that $\sigma_{d} \in R$, we then have that $s \in R$. Therefore, $R=S \cap D^{-1}(K)$.

Finally, to complete the proof, we show that $D(S)$ is $C$-divisible. Let $s \in S$ and $c \in C$. Since $S / A$ is $C$-divisible, there exists $a \in A$ such that $s=a+c \sigma$ for some $\sigma \in S$. Since

$$
\text { Ker } D_{0}=A^{\prime}=\left\{\left\langle a_{c}+c R\right\rangle \in \widetilde{R}: a_{c} \in A \text { for all } c \in C\right\},
$$

it follows that $D(a)=D_{0}(\langle a+c R\rangle)=0$. Hence $D(s)=D(a+c \sigma)=c D(\sigma) \in c D(S)$, which proves that $D(S)$ is $C$-divisible. After replacing $D$ with $\left.D\right|_{S}$, the lemma is proved.

With the aid of Lemma 3.2, we now prove the abstract version of the correspondence theorem to be given in Theorem 4.1 of the next section. Abbreviate $d_{S_{C} / A_{C}}$ by $d$, and for each ring $R$ between $A$ and $S$, define an $S$-submodule of $\Omega_{S_{C} / A_{C}}$ by

$$
\Omega(R):=\sum_{r \in R} S d(r)
$$

that is, $\Omega(R)$ is the $S$-submodule of $\Omega_{S_{C} / A_{C}}$ generated by the image of $R$ under $d$. Our assumptions on $A$ and $S$ imply that $\Omega(S)=\Omega_{S_{C} / A_{C}}$. For since $d$ is $A_{C}$-linear, every element of $\Omega_{S_{C} / A_{C}}$ is of the form $\sum_{i=1}^{n} \frac{s_{i}}{c} d\left(\sigma_{i}\right)$ for some $c \in C$ and $s_{1}, \ldots, s_{n}$, $\sigma_{1}, \ldots, \sigma_{n} \in S$. Since $S / A$ is $C$-divisible, given an element in the above form, we may for each $i=1, \ldots, n$ write $\sigma_{i}=a_{i}+c \tau_{i}$ for some $a_{i} \in A$ and $\tau_{i} \in S$. Thus, since $d$ is $A$-linear, we have

$$
\sum_{i} \frac{s_{i}}{c} d\left(\sigma_{i}\right)=\sum_{i} \frac{s_{i}}{c} d\left(a_{i}+c \tau_{i}\right)=\sum_{i} s_{i} d\left(\tau_{i}\right) \in \Omega(S) .
$$

Therefore, $\Omega(S)=\Omega_{S_{C} / A_{C}}$, and hence $S$ corresponds to $\Omega_{S_{C} / A_{C}}$ under the mappings in the following proposition. 
Proposition 3.3. Let $A \subseteq S$ be an extension of rings, let $C$ be a multiplicatively closed subset of $A$ consisting of nonzerodivisors of $S$, and suppose the inclusion mapping $A \rightarrow S$ is an analytic isomorphism along $C$. Then the mappings

$$
R \mapsto \Omega(R) \text { and } K \mapsto S \cap d_{S_{C} / A_{C}}^{-1}(K)
$$

define a bijection between the intermediate rings $A \subseteq R \subseteq S$ such that $S / R$ is $C$-torsion and $R \subseteq S$ is a quadratic extension, and the set of $S$-submodules $K$ of $\Omega(S)$ such that $\Omega(S) / K$ is $C$-torsion.

Proof. Let $d=d_{S_{C} / A_{C}}$. First, we show these mapping are well defined. Let $K$ be an $S$-submodule of $\Omega(S)$ such that $\Omega(S) / K$ is $C$-torsion, and let $R=S \cap d^{-1}(K)$. Then $R$ is a ring, so we need only show that $R \subseteq S$ is a quadratic extension and $S / R$ is $C$-torsion. First, if $s \in S$, then since $\Omega(S) / K$ is $C$-torsion, there exists a nonzerodivisor $c \in C$ such that $c d(s) \in K$. Since $c \in A$, then $d(c s)=c d(s) \in K$, so that $c s \in S \cap d^{-1}(K)=R$. Hence $S / R$ is $C$-torsion. Next, to prove that $R \subseteq S$ is a quadratic extension we show that (2) of Lemma 3.1 can be substantiated by $R$ and $S$. An appeal to Lemma 3.1 is possible here because $S / R$ is, as we have just seen, $C$-torsion, and since $S / R$ is an $A$-homomorphic image of the $C$-divisible $A$-module $S / A$, the $R$-module $S / R$ is $C$-divisible, and thus the hypotheses of Lemma 3.1 are satisfied. Now, to verify (2) of the lemma, define a mapping

$$
D: S \rightarrow \Omega(S) / K: s \mapsto d(s)+K,
$$

where $s \in S$. Then since $d$ is a derivation, so is $D$ (note that $\Omega(S) / K$ is an $S$-module, since both $\Omega(S)$ and $K$ are). Moreover:

$$
\text { Ker } D=\{s \in S: d(s) \in K\}=S \cap d^{-1}(K)=R .
$$

Hence by Lemma 3.1, $R \subseteq S$ is a quadratic extension.

Conversely, suppose that $A \subseteq R \subseteq S, R \subseteq S$ is a quadratic extension and $S / R$ is $C$-torsion. We claim that $\Omega(S) / \Omega(R)$ is $C$-torsion. Let $x \in \Omega(S)$ and write $x=\sum_{i=1}^{n} s_{i} d\left(\sigma_{i}\right)$ for some $s_{1}, \ldots, s_{n}, \sigma_{1}, \ldots, \sigma_{n} \in S$. Since $S / R$ is $C$-torsion we may choose $c \in C$ such that $c \sigma_{i} \in R$ for all $i=1, \ldots, n$. Then $c x=\sum_{i=1}^{n} s_{i} d\left(c \sigma_{i}\right) \in$ $\Omega(R)$, and this proves that $\Omega(S) / \Omega(R)$ is $C$-torsion. Therefore, the mappings in the theorem are well defined.

To see that $K \mapsto S \cap d^{-1}(K)$ is one-to-one, suppose that $K$ and $K^{\prime}$ are $S$ submodules of $\Omega(S)$ such that $\Omega(S) / K$ and $\Omega(S) / K^{\prime}$ are $C$-torsion and $K \neq K^{\prime}$. We show that $S \cap d^{-1}(K) \neq S \cap d^{-1}\left(K^{\prime}\right)$. Without loss of generality, suppose that there exists $k \in K \backslash K^{\prime}$, and write $k=\sum_{i=1}^{n} s_{i} d\left(x_{i}\right)$ for some $s_{1}, \ldots, s_{n}, x_{1}, \ldots, x_{n} \in S$. Since $\Omega(S) /\left(K \cap K^{\prime}\right)$ is $C$-torsion, there exists $c \in C$ such that $c d\left(x_{i}\right) \in K \cap K^{\prime}$ for all $i=1, \ldots, n$. Also, since $S / A$ is $C$-divisible, there exist for each $i, a_{i} \in A$ and $\sigma_{i} \in S$ such that $s_{i}=a_{i}+c \sigma_{i}$. Therefore, we have:

$$
k=\sum_{i} s_{i} d\left(x_{i}\right)=d\left(\sum_{i} a_{i} x_{i}\right)+\sum_{i} \sigma_{i} c d\left(x_{i}\right) .
$$

By the choice of $c$, it is the case that $\sum_{i} \sigma_{i} c d\left(x_{i}\right) \in K \cap K^{\prime}$. Thus, since $k \notin K^{\prime}$, it must be that $d\left(\sum_{i} a_{i} x_{i}\right) \notin K^{\prime}$. However, $d\left(\sum_{i} a_{i} x_{i}\right)=k-\sum_{i} \sigma_{i} c d\left(x_{i}\right) \in K$, so $\sum_{i} a_{i} x_{i}$ is in $S \cap d^{-1}(K)$ but not in $S \cap d^{-1}\left(K^{\prime}\right)$. This proves that the mapping $K \mapsto S \cap d^{-1}(K)$ is one-to-one.

Next we show that the mapping $K \mapsto S \cap d^{-1}(K)$ maps onto the set of rings $R$ such that $A \subseteq R \subseteq S, R \subseteq S$ is a quadratic extension and $S / R$ is $C$-torsion. Suppose that $R$ is such a ring. We claim that there exists an $S$-submodule $K$ of 
$\Omega(S)$ such that $\Omega(S) / K$ is $C$-torsion and $R=S \cap d^{-1}(K)$. By Lemma 3.2 there exists a $C$-linear derivation $D: S \rightarrow L$ such that $L$ is a $C$-torsion-free $S$-module, $D(S)=c D(S)$ for each $c \in C$, and $R=D^{-1}(N)$ for some $S$-submodule $N$ of $L$ such that $L / N$ is $C$-torsion. By extending $D$ via the quotient rule, we may in fact view $D$ as a derivation from $S_{C}$ to $L_{C}$, so that $R=S \cap D^{-1}(N)$. Now, as we have shown above, $\Omega(S)=\Omega_{S_{C} / A_{C}}$, so by the universal property of the module of Kähler differentials (2.1), there exists an $S$-module homomorphism $f: \Omega(S) \rightarrow L_{C}$ such that $D=f \circ d$. Let $K=f^{-1}(N)$. We claim that $R=S \cap d^{-1}(K)$. Indeed, suppose that $s \in S$ with $d(s) \in K$. Then $D(s)=f(d(s)) \in f(K) \subseteq N$, so that $s \in S \cap D^{-1}(N)=R$. Therefore, $S \cap d^{-1}(K) \subseteq R$. The reverse inclusion is clear, since $D(R) \subseteq N$ and $D=f \circ d$ imply that $d(R) \subseteq f^{-1}(N)=K$. Hence $R=S \cap d^{-1}(K)$. Finally, to see that $\Omega(S) / K$ is $C$-torsion, let $y \in \Omega(S)$. Then $f(y) \in L_{C}$, so since $L / N$ is $C$-torsion, there exists $c \in C$ such that $c f(y) \in N$, and hence $c y \in f^{-1}(N)=K$.

The module $\Omega(R)$ plays a role similar to that of the module of Kähler differentials in that it shares some universal properties with this module:

Lemma 3.4. Let $A \subseteq S$ be an extension of rings, let $C$ be a multiplicatively closed subset of $A$ consisting of nonzerodivisors of $S$, and suppose the inclusion mapping $A \rightarrow S$ is an analytic isomorphism along $C$. Let $L$ be an $S_{C}$-module, and suppose that there is an $A_{C}$-linear derivation $D: S_{C} \rightarrow L$ such that $D\left(S_{C}\right)$ generates $L$ as an $S_{C}$-module. There exists a surjective $S_{C}$-module homomorphism $\alpha: \Omega_{S_{C} / A_{C}} \rightarrow L$ such that $D=\alpha \circ d_{S_{C} / A_{C}}$ and for each $S$-submodule $K$ of $L$ with $K_{C}=L$, then with $R=S \cap D^{-1}(K)$, it is the case that $\alpha(\Omega(R))=K, \Omega(R)=\alpha^{-1}(K)$ and $\alpha$ restricted to $\Omega(R)$ is an analytic isomorphism along $C$.

Proof. Let $d=d_{S_{C} / A_{C}}$. Since $D: S_{C} \rightarrow L$ is an $A_{C}$-linear derivation and (as noted earlier) $\Omega(S)=\Omega_{S_{C} / A_{C}}$, then there exists an $S_{C}$-module homomorphism $\alpha: \Omega(S) \rightarrow L$ such that $D=\alpha \circ d$; see (2.1). Since the image of $D$ generates $L$, it follows that $\alpha$ is surjective. Let $K$ be an $S$-submodule of $L$ with $K_{C}=L$, and let $R=S \cap D^{-1}(K)$. We claim first that $\Omega(R)=\alpha^{-1}(K)$. Let $x \in \Omega(R)$, and write $x=\sum_{i=1}^{n} s_{i} d\left(r_{i}\right)$, where $s_{1}, \ldots, s_{n} \in S$ and $r_{1}, \ldots, r_{n} \in R$. Then

$$
\alpha(x)=\sum_{i} s_{i} \alpha\left(d\left(r_{i}\right)\right)=\sum_{i} s_{i} D\left(r_{i}\right) \in K,
$$

and this shows that $\Omega(R) \subseteq \alpha^{-1}(K)$. To see that the reverse inclusion holds, let $y \in \alpha^{-1}(K)$ and write $y=\sum_{i=1}^{n} \sigma_{i} d\left(\tau_{i}\right)$ for some $\sigma_{1}, \ldots, \sigma_{n}, \tau_{1}, \ldots, \tau_{n} \in S$. Since $S / R$ is $C$-torsion, there exists $c \in C$ such that $c \tau_{i} \in R$ for all $i$. Also, since $S / A$ is $C$-divisible, for each $i$ there exist $a_{i} \in A$ and $u_{i} \in S$ such that $\sigma_{i}=a_{i}+c u_{i}$. Then

$$
y=\sum_{i} \sigma_{i} d\left(\tau_{i}\right)=d\left(\sum_{i} a_{i} \tau_{i}\right)+\sum_{i} u_{i} d\left(c \tau_{i}\right) .
$$

By assumption $\alpha(y) \in K$, so, since $D=\alpha \circ d$, we have

$$
D\left(\sum_{i} a_{i} \tau_{i}\right)+\sum_{i} u_{i} D\left(c \tau_{i}\right) \in K \text {. }
$$

Now, since for each $i, c \tau_{i} \in R=D^{-1}(K) \cap S$, it follows that $\sum_{i} u_{i} D\left(c \tau_{i}\right) \in K$. Therefore, $D\left(\sum_{i} a_{i} \tau_{i}\right) \in K$, which shows that $\sum_{i} a_{i} \tau_{i} \in S \cap D^{-1}(K)=R$. Thus

$$
y=d\left(\sum_{i} a_{i} \tau_{i}\right)+\sum_{i} u_{i} d\left(c \tau_{i}\right) \in \Omega(R)
$$


and this proves that $\Omega(R)=\alpha^{-1}(K)$. Moreover, from the fact that $\alpha(\Omega(S))=K_{C}$ and $\alpha^{-1}(K)=\Omega(R)$, it follows that $\alpha(\Omega(R))=K$.

Finally, to prove that $\alpha$ is an analytic isomorphism, note first that for each $c \in C$, $\alpha$ induces the mapping

$$
\alpha_{c}: \Omega(R) / c \Omega(R) \rightarrow K / c K: x+c \Omega(R) \mapsto \alpha(x)+c K,
$$

where $x \in K$. This mapping is clearly a well-defined $S$-module homomorphism, and since $\alpha$ is onto, so is $\alpha_{c}$. To see that it is injective, suppose $x \in \Omega(R)$ such that $\alpha(x) \in c K$. Since $x \in \Omega(R)$, there exist $s_{1}, \ldots, s_{n} \in S$ and $r_{1}, \ldots, r_{n} \in R$ such that $x=\sum_{i} s_{i} d\left(r_{i}\right)$. For each $i$, write $s_{i}=a_{i}+c \sigma_{i}$, where $a_{i} \in A$ and $\sigma_{i} \in S$. Then

$$
x=d\left(\sum_{i} a_{i} r_{i}\right)+c \sum_{i} \sigma_{i} d\left(r_{i}\right) .
$$

Set $r=\sum_{i} a_{i} r_{i}$ and $y=\sum_{i} \sigma_{i} d\left(r_{i}\right)$, so that $x=d(r)+c y$, with $y \in \Omega(R)$. Then using the fact that $D=\alpha \circ d$, it follows that $D(r)+c \alpha(y)=\alpha(x) \in c K$. Since $y \in \Omega(R)$ and $\alpha(\Omega(R))=K$, we have $c \alpha(y) \in c K$. Hence $D(r) \in c K$. Now since $S / A$ is $C$-divisible, we may write $r=a+c s$ for some $a \in A$ and $s \in S$. Thus, since $D$ is $A$-linear, $c D(s)=D(c s)=D(a+c s)=D(r) \in c K$, and since $L=K_{C}$ is $C$-torsion-free, $D(s) \in K$. But then $s \in S \cap D^{-1}(K)=R$, so $d(r)=d(a+c s)=c d(s) \in c \Omega(R)$. Therefore, $x=d(r)+c y \in c \Omega(R)$, and this proves that $\alpha_{c}$ is injective. Hence $\alpha_{c}$ is an isomorphism of $S$-modules.

The next proposition will be useful in Section 4 for describing the completion of bad stable rings in immediate extensions of DVRs.

Proposition 3.5. Let $A \subseteq S$ be an extension of rings, let $C$ be a multiplicatively closed subset of $A$ consisting of nonzerodivisors of $S$, and suppose the inclusion mapping $A \rightarrow S$ is an analytic isomorphism along $C$. Let $K$ be a $C$-torsion-free $S$ module, and suppose that there is an $A_{C}$-linear derivation $D: S_{C} \rightarrow K_{C}$ such that $D\left(S_{C}\right)$ generates $K_{C}$ as an $S_{C}$-module. Let $R=S \cap D^{-1}(K)$. Then the mapping

$$
f: R \rightarrow S \star K: r \mapsto(r, D(r))
$$

is an analytic isomorphism along $C$.

Proof. We prove the proposition by verifying three claims.

Claim 1. For each $c \in C, K=D(c S \cap R)+c K$.

Let $c \in C$, and let $k \in K$. Then by Lemma 3.4, there exists a surjective $S$-module homomorphism $\alpha: \Omega_{S_{C} / A_{C}} \rightarrow K_{C}$ such that $D=\alpha \circ d_{S_{C} / A_{C}}$ and $\alpha(\Omega(R))=K$. Thus $k=\sum_{i=1}^{n} s_{i} D\left(r_{i}\right)$, for some $r_{i} \in R$ and $s_{i} \in S$. Since $S / A$ is $C$-divisible, for each $i$, we may write $s_{i}=a_{i}+c \sigma_{i}$ for some $a_{i} \in A$ and $\sigma_{i} \in S$. Thus, setting $r=\sum_{i} a_{i} r_{i}$, we have $k=D(r)+\sum_{i} c \sigma_{i} D\left(r_{i}\right)$. Similarly, we may write $r=a+c \sigma$ for some $a \in A$ and $\sigma \in S$, so that $c \sigma \in c S \cap R$. Thus, since $D$ is $A$-linear, $D(r)=D(c \sigma)$, and we have that $k=D(c \sigma)+\sum_{i} c \sigma_{i} D\left(r_{i}\right)$. Hence $k \in D(c S \cap R)+c K$, which verifies that $K=D(c S \cap R)+c K$.

Claim 2. For each $c \in C, c S \cap D^{-1}(c K)=c R$.

Let $c \in C$ and $s \in S$ such that $D(c s) \in c K$. Then $c D(s)=D(c s) \in c K$. Hence, since $K$ is $C$-torsion-free, $D(s) \in K$. But $R=S \cap D^{-1}(K)$, so $s \in R$. This shows that $c S \cap D^{-1}(c K)=c R$. 
Claim 3. $f$ is an analytic isomorphism along $C$.

It is enough to show that the cokernel of $f$ is $C$-torsion-free and $C$-divisible. First we show that the cokernel of $f$ is $C$-torsion-free. Suppose that $s \in S, k \in K$, and $c \in C$ such that $c \cdot(s, k)=(r, D(r))$ for some $r \in R$. Then $D(c s) \in c K$, so that $c s \in c S \cap D^{-1}(c K)$. Hence by Claim 2 and the fact that $c$ is a nonzerodivisor in $S$, $s \in R$. Also, since $c D(s)=D(c s)=c k$ and $K$ is $C$-torsion-free, we have $D(s)=k$, which proves that $(s, k)=(s, D(s)) \in f(R)$.

Next we show that the cokernel of $f$ is $C$-divisible. Let $c \in C$ and $k \in K$. We claim that $(0, k) \in f(R)+c \cdot(S \star K)$. By Claim 1 we may write $k=D(c \sigma)+c k^{\prime}$ for some $k^{\prime} \in K$ and $\sigma \in S$ such that $c \sigma \in R$. Now

$$
(0, k)=(c \sigma, D(c \sigma))+c \cdot\left(-\sigma, k^{\prime}\right) \in f(R)+c \cdot(S \star K) .
$$

Thus to complete the proof that the cokernel of $f$ is $C$-divisible, it suffices to show that for each $s \in S,(s, 0) \in f(R)+c \cdot(S \star K)$.

Let $s \in S$. Since $S / A$ is $C$-divisible, then $S=A+c S$, and we may write $s=a+c \sigma$, where $\sigma \in S$ and $a \in A$. Therefore, since $D(a)=0$, we have

$$
(s, 0)=(a, D(a))+(c \sigma, 0) \in f(R)+c \cdot(S \star K),
$$

and we conclude that the cokernel of $f$ is $C$-divisible.

Corollary 3.6. Let $A \subseteq R \subseteq S$ be an extension of rings, let $C$ be a multiplicatively closed subset of $A$ consisting of nonzerodivisors of $S$, and suppose the inclusion mapping $A \rightarrow S$ is an analytic isomorphism along $C$. If $R \subseteq S$ is a quadratic extension and $S / R$ is $C$-torsion, then the mapping

$$
f: R \rightarrow S \star \Omega(R): r \mapsto(r, d(r))
$$

is an analytic isomorphism along $C$.

Proof. The $S$-module $\Omega(R)$ is generated by $d_{S_{C} / A_{C}}(R)$, and by Proposition 3.3 . $R=S \cap d_{S_{C} / A_{C}}^{-1}(\Omega(R))$, so the corollary follows from Proposition 3.5 .

\section{Stable Rings Between DVRs}

We apply the results of the previous section on analytic isomorphisms to our main case of interest, where $A \subseteq S$ is an immediate extension of DVRs. It is straightforward to check that the inclusion mapping of an immediate extension $U \subseteq V$ of DVRs is an analytic isomorphism along the multiplicatively closed set $C=U \backslash\{0\}$ and that $U_{C}$ and $V_{C}$ are the quotient fields of $U$ and $V$, respectively, so all of the results of the previous section can be translated into the present context. Let $Q$ and $F$ denote the quotient fields of $U$ and $V$, respectively. We consider rings $R$ between $U$ and $V$, and as in the last section we associate to each such ring the $V$-submodule $\Omega(R)$ of $\Omega_{F / Q}$ generated by $d_{F / Q}(R)$; that is,

$$
\Omega(R):=\sum_{r \in R} V d_{F / Q}(r) .
$$

The correspondence in Proposition 3.3 now translates into the form given in Theorem 4.1. Recall that when $L$ is a torsion-free module over a domain, then a submodule $K$ of $L$ is full if $L / K$ is a torsion module. 
Theorem 4.1. Let $U \subseteq V$ be an immediate extension of DVRs having quotient fields $Q$ and $F$, respectively. There is a one-to-one correspondence between bad stable rings $R$ with $U \subseteq R \subseteq V$ and normalization $V$, and proper full $V$-submodules $K$ of $\Omega_{F / Q}$ given by

$$
R \mapsto \Omega(R) \text { and } K \mapsto V \cap d_{F / Q}^{-1}(K) .
$$

Proof. In light of Proposition 3.3, with $C=U \backslash\{0\}$, all that needs to be verified for the correspondence is that a ring $R$ between $U$ and $V$ is stable with normalization $V$ if and only if $R \subseteq V$ is a quadratic extension and $V / R$ is a torsion $U$-module. If $R$ is stable with normalization $V$, then $V / R$ is a torsion $R$-module. Since $R$ has dimension one and dominates $U$, it follows that $Q R=F$, and hence $V / R$ is torsion not just as an $R$-module, but as a $U$-module also. Moreover, by (2.4), $R \subseteq V$ is a quadratic extension. Conversely, if $V / R$ is a torsion $U$-module and $R \subseteq V$ is a quadratic extension, then $R$ and $V$ share the same quotient field $F$, and since $R \subseteq V$ is an integral extension and $V$ is a DVR, it follows that $V$ is the normalization of $R$. Hence by (2.4), $R$ is either equal to $V$ or is a bad stable ring.

The theorem makes it possible to pinpoint when there is an analytically ramified local Noetherian $U$-subalgebra of $V$ having normalization $V$. To prove this we recall a fact due to Matlis that every one-dimensional analytically ramified local Noetherian domain has a bad 2-generator overring [18, Theorem 14.16]. This result is proved from a different point of view in [25, Theorem 5.10]. In fact, for the purposes of the corollary, Theorem 5.1 below would also suffice.

Corollary 4.2. Let $U \subseteq V$ be an immediate extension of DVRs with quotient fields $Q$ and $F$, respectively. Then the following are equivalent:

(1) There exists an analytically ramified local Noetherian domain containing $U$ and having normalization $V$.

(2) Either $F$ has characteristic 0 and $F / Q$ is not algebraic, or $F$ has characteristic $p>0$ and $F \neq Q\left[F^{p}\right]$.

Proof. If there exists an analytically ramified local Noetherian domain $A$ containing $U$ and having normalization $V$, then as discussed before the corollary, there exists a bad stable ring $R$ containing $A$ and having normalization $V$. Thus by Theorem 4.1, $\Omega_{F / Q} \neq 0$. By (2.2), the nonvanishing of $\Omega_{F / Q}$ is equivalent to (2) being valid for $F / Q$. Therefore, by Theorem 4.1, to prove the converse it suffices to note that the nonzero $F$-vector space $\Omega_{F / Q}$ contains a proper full $V$-submodule.

Remark 4.3. The idea of constructing analytically ramified local Noetherian domains using derivations originates with Ferrand and Raynaud in 6]. Their method, along with a generalization by Goodearl and Lenagan [8, motivates Theorem 4.1. although our approach and results differ. The connection with the articles [6] and [8] is more evident in [24].

Also to $R$ we associate the following cardinal number, which did not have an analogue in the last section:

$$
\epsilon_{R}:=\operatorname{dim}_{V / \mathfrak{M}_{V}} \Omega(R) / \mathfrak{M}_{V} \Omega(R) .
$$

We see in the next theorem that this cardinal is one less than the embedding dimension of $R$. In the theorem, we deduce from Corollary 3.6 a description of the completion of bad stable rings between $U$ and $V$. Recall from (2.5) that the $M$-adic 
completion $\widehat{R}$ of a bad stable ring $R$ has a prime ideal $P$ such that $P^{2}=0$ and $\widehat{R} / P$ is a DVR. Theorem 4.4 shows that $P$ splits $\widehat{R}$, making $\widehat{R}$ an idealization. In the theorem, $\widehat{R}$ denotes the completion of $R$ in the $M$-adic topology, where $M$ is the maximal ideal of $R$, while $\widehat{V}$ and $\widehat{\Omega}(R)$ denote the completion of $V$ and $\Omega(R)$ in the $\mathfrak{M}_{V}$-adic topology (which since $M$ is stable and $V$ is a DVR is easily seen to coincide with the $M$-adic topology).

Theorem 4.4. Let $U \subseteq V$ be an immediate extension of DVRs having quotient fields $Q$ and $F$, respectively, and let $R$ be a stable ring between $U$ and $V$ with normalization $V$. Then the following statements hold for $R$ :

(1) The mapping $f$ defined by

$$
f: R \rightarrow V \star \Omega(R): r \mapsto\left(r, d_{F / Q}(r)\right)
$$

lifts to an isomorphism of rings:

$$
\widehat{R} \rightarrow \widehat{V} \star \widehat{\Omega}(R) .
$$

(2) $R$ is Noetherian (with embedding dimension $\epsilon_{R}+1$ ) if and only if $\epsilon_{R}$ is finite.

(3) The dimension of the F-vector space $\Omega_{F / Q}$ is at least $\epsilon_{R}$.

Proof. (1) Observe that $V / R$ is $C$-torsion for $C=U \backslash\{0\}$ since $F=Q V=Q R$. Thus by Corollary 3.6, for each $0 \neq c \in U$, the induced mapping

$$
f_{c}: R / c R \rightarrow V / c V \star \Omega(R) / c \Omega(R)
$$

is an isomorphism of $U$-algebras. A straightforward verification using the fact that $d_{F / Q}$ is $U$-linear then shows that the mapping $f_{c}$ induces an isomorphism of rings:

$$
\lim _{\longleftarrow} R / c R \rightarrow\left(\lim _{\longleftarrow} V / c V\right) \star\left(\lim _{\longleftarrow} \Omega(R) / c \Omega(R)\right),
$$

where the inverse limits range over all $0 \neq c \in U$. Since $R$ is stable, the maximal ideal $M$ of $R$ has the property that $M^{2}=m M$ for some $m \in M$. Since $R$ is one-dimensional, then $\sqrt{m R}=\sqrt{c R}=M$ for any choice of $0 \neq c \in \mathfrak{M}_{U}$. Moreover, since $M^{2} \subseteq m R \subseteq M$, it follows that for each $0 \neq c \in \mathfrak{M}_{U}$, there is a power of $M$ contained in $c \bar{R}$. Therefore, $\widehat{R}$ is isomorphic to $\lim R / c R$. Also, since $U \subseteq V$ is an immediate extension, it follows that $\widehat{V}$ is isomorphic to $\lim V / c V$ and $\widehat{\Omega}(R)$ is isomorphic to $\lim _{\longleftarrow} \Omega(R) / c \Omega(R)$. Therefore, since all the maps involved are natural, $f$ lifts to the isomorphism in the theorem.

(2) Let $K=\Omega(R)$. By Corollary 3.6, the mapping $f_{c}: R / c R \rightarrow V / c V \star K / c K$ is an isomorphism for each $0 \neq c \in U$. Since $R$ has dimension 1 and the maximal ideal of $R$ contains $\mathfrak{M}_{U}$, every principal ideal of $R$ contains a power of some nonzero element of $U$. From this it follows that $R$ is a Noetherian domain if and only if for each $0 \neq c \in U, K / c K$ is a finitely generated $V$-module. Thus if $R$ is a Noetherian domain, it must be that $K / \mathfrak{M}_{V} K$ is a finitely generated $V$-module, and hence its dimension $\epsilon_{R}$ as a $V / \mathfrak{M}_{V}$-vector space is finite. Conversely, if $K / \mathfrak{M}_{V} K$ has finite dimension as a $V / \mathfrak{M}_{V}$-vector space and $0 \neq c \in \mathfrak{M}_{U}$, then since $\mathfrak{M}_{V}^{k}=c V$ for some $k>0$, it follows that $K / c K$ is a finitely generated $V$-module. Thus if $\epsilon_{R}$ is finite, then $R$ is Noetherian. The assertion about embedding dimension now follows from (1) and the fact that finitely generated modules over DVRs are free. Indeed, by 
the theorem, $\widehat{R} \cong \widehat{V} \star \widehat{\Omega}(R)$, and when $R$ is Noetherian, so is $\widehat{R}$, so that $\widehat{\Omega}(R)$ is a finitely generated free $\widehat{V}$-module. Its rank is $\epsilon_{R}$, so the embedding dimension of $\widehat{R}$ is one more than this rank.

(3) Let $\mathfrak{M}=\mathfrak{M}_{V}, K=\Omega_{F / Q}$ and $d=d_{F / Q}$. A consequence of Claim 1 in the proof of Proposition 3.5 is that $K=d(R)+\mathfrak{M} K$. Thus we may choose a collection $\mathcal{B}$ of elements of $R$ such that $\{d(r): r \in \mathcal{B}\}$ consists of representatives of basis elements of the $V / \mathfrak{M}$-vector space $K / \mathfrak{M} K$. To prove (3) then, it suffices to show that the elements $d(r), r \in \mathcal{B}$, are linearly independent elements of the $F$-vector space $\Omega_{F / Q}$. To this end, suppose that $r_{1}, \ldots, r_{m} \in \mathcal{B}$ and $s_{1}, \ldots, s_{m} \in F$ such that $s_{1} d\left(r_{1}\right)+\cdots+s_{m} d\left(r_{m}\right)=0$. By clearing denominators, we may assume that each $s_{i} \in V$. Moreover, if each $s_{i} \in \mathfrak{M}$, then we may divide by the generator of the principal ideal $\mathfrak{M}$ of the DVR $V$ until some $s_{i}$, say $s_{1}$, is in $V \backslash \mathfrak{M}$. But then $s_{1}+\mathfrak{M}$ is a nonzero coefficient of $d\left(r_{1}\right)+\mathfrak{M} K$ in the equation

$$
\sum_{i=1}^{m}\left(s_{i}+\mathfrak{M}\right)\left(d\left(r_{i}\right)+\mathfrak{M} K\right)=0+\mathfrak{M} K,
$$

which forces the images of $d\left(r_{1}\right), \ldots, d\left(r_{m}\right)$ to be linearly dependent in the $V / \mathfrak{M}$ vector space $K / \mathfrak{M} K$. This contradiction proves that $\epsilon_{R} \leq \operatorname{dim}_{F} \Omega_{F / Q}$.

\section{LOCAL RINGS BETWEEN DVRS}

In the last section we considered stable rings in an immediate extension of DVRs. In this section, we consider extensions $U \subseteq A \subseteq V$ where $U \subseteq V$ is an immediate extension of DVRs and $A$ is a local Noetherian domain birationally dominated by $V$, and in this more nuanced setting we describe the bad stable rings between $A$ and $V$. To illustrate how such a setting can occur, consider a local Noetherian domain $A$ containing a field $k$ such that $A=k+\mathfrak{m}$, where $\mathfrak{m}$ is the maximal ideal of $A$. Then straightforward arguments show that there exist DVRs $U$ and $V$ such that $U \subseteq A \subseteq V$ and $V$ birationally dominates $A$ if and only if there is an embedding $f: A \rightarrow k[[T]]$, where $T$ is an indeterminate for $k$, such that $\mathfrak{m} k[[T]]=T k[[T]]$. Geometrically, the existence of such an embedding $f$ can be expressed as saying that there is a fat, nonsingular $k$-arc $\operatorname{Spec}(k[[T]]) \rightarrow \operatorname{Spec}(A)$; see for example [12].

Returning to the abstract setting $U \subseteq A \subseteq V$, recall that a quasilocal domain $B$ birationally dominates $A$ if $A \subseteq B, A$ and $B$ have the same quotient field, and the maximal ideal of $A$ is contained in the maximal ideal of $B$. Using the subring $U$ of $A$, we describe the smallest bad stable ring between $A$ and $V$. This provides a somewhat natural source of one-dimensional analytically ramified Noetherian domains birationally dominating local Noetherian domains.

Our focus in this section is on a specific ring between $A$ and $V$, the ring given by $R=\operatorname{Ker} d_{V / A}$, where $d_{V / A}: V \rightarrow \Omega_{V / A}$ is the exterior differential of the ring extension $V / A$. Since $d_{V / A}$ is a derivation, $R=\operatorname{Ker} d_{V / A}$ is a ring between $A$ and $V$. Our interest in this ring lies in the fact that it is the smallest bad stable ring between $A$ and $V$. More precisely, in [25, Theorem 5.9] it is shown that if $B$ is a local Noetherian domain with maximal ideal $\mathfrak{m}$ and $W$ is a DVR which birationally dominates $B$ and has the property that $W=B+\mathfrak{m} W$ (in which case we say that $W$ tightly dominates $B$ ), then the ring $\operatorname{Ker} d_{W / B}$ is a bad stable ring that is contained in every stable ring between $B$ and $W$; equivalently, this kernel is the smallest ring between $B$ and $W$ which forms a quadratic extension with $W$. It is not clear whether in the general context just mentioned for $B$ that the kernel must always be 
a Noetherian ring. However, in the setting of this article, it is not only Noetherian, but its maximal ideal is extended from that of the base ring:

Theorem 5.1. Let $U \subseteq A \subsetneq V$ be an extension of local Noetherian domains, where $U \subseteq V$ is an immediate extension of DVRs and $V$ birationally dominates $A$. The ring $R=\operatorname{Ker} d_{V / A}$ is a bad Noetherian stable ring that tightly dominates $A, R$ has normalization $V, R$ has maximal ideal $\mathfrak{m} R$ extended from the maximal ideal $\mathfrak{m}$ of $A$, and $R$ is contained in every stable ring between $A$ and $V$.

Proof. Observe that $\mathfrak{m}=\mathfrak{M}_{V} \cap A$, since $V$ dominates $A$. Since $U \subseteq V$ is an immediate extension, $\mathfrak{M}_{U}=\mathfrak{M}_{V} \cap U$, and hence $\mathfrak{M}_{U}=\mathfrak{m} \cap U$. The maximal ideal of $U$ extends to the maximal ideal of $V$, and hence $\mathfrak{M}_{V}=\mathfrak{m} V$. Also from the immediacy of the extension, $V=U+\mathfrak{m} V$, so that necessarily $V=A+\mathfrak{m} V$. Therefore, $V$ tightly dominates $A$, and hence as noted before the theorem, $R$ is a bad stable domain with normalization $V$ that is contained in every stable ring between $A$ and $V$. Thus it remains to show that $R$ tightly dominates $A$ and $R$ is Noetherian with maximal ideal $\mathfrak{m} R$. Since $R$ has Krull dimension 1 and $\mathfrak{m}$ is a finitely generated ideal of $A$, it suffices to prove that $R=A+\mathfrak{m} R$. But from $V=A+\mathfrak{m} V$ we deduce that $R=A+(\mathfrak{m} V \cap R)$, and hence the residue field of $R$ is isomorphic to that of $A$. Therefore, all that remains to be proved is that $\mathfrak{m} R$ is the maximal ideal of $R$.

We claim that $\Omega(R)=\Omega(A)$, and we prove first that $\Omega(A)$ is a full $V$-submodule of $\Omega_{F / Q}$, where $Q$ and $F$ are the quotient fields of $U$ and $V$, respectively. To simplify notation let $d=d_{F / Q}$. Let $f \in F$. Then $f=\frac{a}{b}$ for some $a, b \in A$, so by the quotient rule,

$$
d(f)=\frac{a \cdot d(b)-b \cdot d(a)}{b^{2}}
$$

and hence $b^{2} \cdot d(f) \in \Omega(A)$. Since $\Omega_{F / Q}$ is generated as an $F$-vector space by elements of the form $d(f)$, it follows that $\Omega(A)$ is a full $V$-submodule of $\Omega_{F / Q}$.

Next we show that $R=V \cap d^{-1}(\Omega(A))$. Let $T=V \cap d^{-1}(\Omega(A))$, so that the claim is then that $R=T$. By Theorem 4.1, either $T=V$ or $T$ is a bad stable domain with normalization $V$. Either way we have by the minimality of $R$ among the class of stable rings between $A$ and $V$ that $R \subseteq T$, so we need only argue the reverse inclusion that $T \subseteq R$. Now by Theorem 4.1, $R=V \cap d^{-1}(\Omega(R))$. But since clearly $\Omega(A) \subseteq \Omega(R)$, we see that

$$
T=V \cap d^{-1}(\Omega(A)) \subseteq V \cap d^{-1}(\Omega(R))=R .
$$

This proves that $R=T$. In fact, since $\Omega(R)$ and $\Omega(A)$ are full $V$-submodules of $\Omega_{F / Q}$, and as we have just shown, $V \cap d^{-1}(\Omega(A))=V \cap d^{-1}(\Omega(R))$, then from Theorem 4.1 we conclude that $\Omega(A)=\Omega(R)$.

Having established that $R=V \cap d^{-1}(\Omega(A))$, we now use this fact to prove that $R$ has maximal ideal $\mathfrak{m} R$. Since $V$ is a DVR with maximal ideal $\mathfrak{M}_{V}=\mathfrak{m} V$ and the maximal ideal $\mathfrak{m} \cap U$ of $U$ extends to $\mathfrak{m} V$ (here we are using the immediacy of the extension $U \subseteq V$ ), there exists $t \in \mathfrak{m} \cap U$ such that $\mathfrak{M}_{U}=t U$ and $\mathfrak{M}_{V}=t V$. By Theorem 4.4(1) there is an isomorphism of $U$-algebras

$$
R / t R \rightarrow V / t V \star \Omega(A) / t \Omega(A) .
$$

As such, this isomorphism carries the maximal ideal of $R / t R$ to the maximal ideal of the image, and so induces the isomorphism

$$
\alpha: M / t R \rightarrow M V / t V \star \Omega(A) / t \Omega(A): m+t R \rightarrow(m+t V, d(m)+t \Omega(A)) .
$$


Since $t V \subseteq M V \subsetneq V$ (the properness of the last inclusion is a consequence of the fact that $R \subseteq V$ is integral) and $\mathfrak{m} V=t V$ is the maximal ideal of $V$, it must be that $M V=t V$. Therefore, the mapping $\alpha$ induces an isomorphism of $U$-modules:

$$
\beta: M / t R \rightarrow \Omega(A) / t \Omega(A): m+t R \mapsto d(m)+t \Omega(A) .
$$

Write $\mathfrak{m}=\left(x_{1}, \ldots, x_{n}\right) A$. We show that $M=\left(t, x_{1}, \ldots, x_{n}\right) R$, hence proving that $M=\mathfrak{m} R$. In light of the isomorphism $\beta$ it suffices to prove that

$$
\Omega(A)=U d\left(x_{1}\right)+\cdots+U d\left(x_{n}\right)+t \Omega(A) .
$$

Moreover, since $V=U+t V$ and $\Omega(A)$ is generated as a $V$-module by the elements $d(b), b \in A$, it is enough to show that for all $b \in A$,

$$
d(b) \in U d\left(x_{1}\right)+\cdots+U d\left(x_{n}\right)+t \Omega(A) .
$$

Let $b \in A$. Since $V=A+\mathfrak{m} V$, then $A / \mathfrak{m} \cong V / \mathfrak{m} V$. But since $U \subseteq V$ is immediate, $U$ and $V$ have the same residue field, and hence $A / \mathfrak{m} \cong V / \mathfrak{m} V \cong U /(\mathfrak{m} \cap U)$. Therefore, $A=U+\mathfrak{m}=U+\left(x_{1}, \ldots, x_{n}\right) A$, and we may write $b=a+x_{1} b_{1}+\cdots+x_{n} b_{n}$ for some $a \in U$ and $b_{i} \in A$. Thus since $d$ is $U$-linear, we have

$$
\begin{aligned}
d(b) & =d\left(x_{1} b_{1}\right)+\cdots+d\left(x_{n} b_{n}\right) \\
& =b_{1} d\left(x_{1}\right)+\cdots+b_{n} d\left(x_{n}\right)+x_{1} d\left(b_{1}\right)+\cdots+x_{n} d\left(b_{n}\right) \\
& \in b_{1} d\left(x_{1}\right)+\cdots+b_{n} d\left(x_{n}\right)+t \Omega(A),
\end{aligned}
$$

where the last assertion is a consequence of the fact that $x_{1}, \ldots, x_{n} \in t V$. Now since $V=U+t V$, we may for each $i$ write $b_{i}=a_{i}+t s_{i}$ for some $a_{i} \in U$ and $s_{i} \in V$. Therefore,

$$
\begin{aligned}
d(b) & \in b_{1} d\left(x_{1}\right)+\cdots+b_{n} d\left(x_{n}\right)+t \Omega(A) \\
& =a_{1} d\left(x_{1}\right)+\cdots+a_{n} d\left(x_{n}\right)+t \Omega(A) \\
& \subseteq U d\left(x_{1}\right)+\cdots+U d\left(x_{n}\right)+t \Omega(A),
\end{aligned}
$$

and this verifies that $M=\mathfrak{m} R$.

In 25], a method due to Heinzer, Rotthaus and Sally from [10 is used in the following way to link stable rings to prime ideals in the generic formal fiber. Let $B$ be a local Noetherian domain tightly dominated by a DVR $W$ and having quotient field $Q(B)$, and let $P$ be the kernel of the canonical mapping $\widehat{B} \rightarrow \widehat{W}$. Then a ring $S$ properly between $B$ and $W$ is a bad Noetherian stable ring tightly dominating $B$ if and only if $S=Q(B) \cap(\widehat{B} / J)$, where $J$ is a $P$-primary ideal of $\widehat{B}$ with $P^{2} \subseteq J \subsetneq P$ [25. Theorem 5.3]. Thus there is a smallest bad stable ring tightly dominating $B$ and having normalization $W$, and it is given by $S=F \cap\left(\widehat{B} / P^{(2)}\right)$, where $P^{(2)}$ is the second symbolic power of $P$. The embedding dimension of this smallest stable ring $S$ is 1 more than the embedding dimension of $\widehat{B}_{P}$ [25, Theorem 5.3].

Thus, returning to our context, we have from Theorem 5.1 that $R=\operatorname{Ker} d_{V / A}$ tightly dominates $A$, and hence this ring falls into the classification above. But since it is by this theorem the smallest bad stable ring between $A$ and $V$ and since it tightly dominates $A$, it must be the ring defined using the ideal $P^{(2)}$. Therefore, also using Theorem 4.4 (2) to calculate embedding dimension, we have the following description of $R=\operatorname{Ker} d_{V / A}$ :

Corollary 5.2. Let $U \subseteq A \subsetneq V$ be an extension of local Noetherian domains, where $U \subseteq V$ is an immediate extension of DVRs and $V$ birationally dominates $A$. 
Let $F$ denote the quotient field of $V$. With $P$ the kernel of the canonical mapping $\widehat{A} \rightarrow \widehat{V}$, the ring $R=$ Ker $d_{V / A}$ can be represented as

$$
R=F \cap\left(\widehat{A} / P^{(2)}\right),
$$

and the embedding dimension of $R$ is given by

$$
\text { emb.dim } R=1+\epsilon_{R}=1+\text { emb.dim } \widehat{A}_{P} \text {. }
$$

We next consider the case where $A$ is essentially of finite type over $U$. In this case the embedding dimension of $R$ depends ultimately only on the field extension $F / Q$, where $Q$ and $F$ are the quotient fields of $U$ and $V$, respectively.

Theorem 5.3. Let $U \subseteq A \subsetneq V$ be an extension of local Noetherian domains, where $U \subseteq V$ is an immediate extension of DVRs with quotient fields $Q$ and $F$, respectively, and $V$ birationally dominates $A$. Let $R=\operatorname{Ker} d_{V / A}$. If $A$ is essentially of finite type over $U$ with Krull dimension $d>1$, then

$$
\text { emb.dim } R=1+\operatorname{dim}_{F} \Omega_{F / Q},
$$

and for $P$ the kernel of $\widehat{A} \rightarrow \widehat{V}$,

$$
\text { emb.dim } \widehat{A}_{P}=\operatorname{dim}_{F} \Omega_{F / Q} .
$$

Proof. Since $A$ is the localization of a finitely generated $U$-subalgebra of $V$ having quotient field $F$, the field $F$ is a finitely generated field extension of $Q$. Moreover, since $A$ has Krull dimension $>1, F$ is not algebraic over $Q$, and thus $\Omega_{F / Q} \neq 0$ (2.2). Also, since the image under $d_{F / Q}$ of any generating set of the field extension $F / Q$ generates the $F$-vector space $\Omega_{F / Q}$, then $\Omega_{F / Q}$ has finite dimension as an $F$-vector space. Write $A=U\left[x_{1}, \ldots, x_{n}\right]_{\mathfrak{p}}$ for some $x_{1}, \ldots, x_{n} \in A$ and prime ideal $\mathfrak{p}$ of $U\left[x_{1}, \ldots, x_{n}\right]$. Let $K$ be a free $V$-submodule of $\Omega_{F / Q}$ such that $\operatorname{rank}(K)=$ $\operatorname{dim}_{F} \Omega_{F / Q}$ and $d_{F / Q}\left(x_{1}\right), \ldots, d_{F / Q}\left(x_{n}\right) \in K$. Set $T=V \cap d_{F / Q}^{-1}(K)$, and observe that since $d_{F / Q}$ is $U$-linear and $d_{F / Q}\left(x_{i}\right) \in K$ for all $i$, it follows that $U\left[x_{1}, \ldots, x_{n}\right] \subseteq$ $T$. Since $V$ birationally dominates $A$, it must be that $\mathfrak{p}=\mathfrak{M}_{V} \cap U\left[x_{1}, \ldots, x_{n}\right]$. Now since $V$ is the normalization of $T$ (Theorem 4.1), $T$ is a quasilocal ring with maximal ideal $\mathfrak{M}_{V} \cap T$. Thus the maximal ideal of $T$ contracts in $U\left[x_{1}, \ldots, x_{n}\right]$ to $\mathfrak{p}$, and we conclude that $A \subseteq T$. By Theorem 4.1, $T \subsetneq V, K=\Omega(T)$ and $T$ is a bad stable ring with normalization $V$. By Theorem 4.4(2),

$$
\text { emb.dim } T=1+\operatorname{dim}_{V / \mathfrak{M}_{V}} K / \mathfrak{M}_{V} K=1+\operatorname{rank}(K)=1+\operatorname{dim}_{F} \Omega_{F / Q} .
$$

Next we consider the ring $R=\operatorname{Ker} d_{V / A}$. By Theorem 5.1, $R$ is a bad stable domain contained in every bad stable ring between $A$ and $V$. In particular, $R \subseteq T$. We claim that emb.dim $R=$ emb.dim $T$. By Theorem 4.4, emb.dim $R=1+\epsilon_{R} \leq$ $1+\operatorname{dim}_{F} \Omega_{F / Q}$. Moreover, since $T$ is between the stable ring $R$ and the normalization of $R$, emb.dim $T \leq$ emb.dim $R$ [25, Theorem 4.4]. Therefore,

$$
1+\operatorname{dim}_{F} \Omega_{F / Q}=\text { emb.dim } T \leq \text { emb.dim } R \leq 1+\operatorname{dim}_{F} \Omega_{F / Q},
$$

and hence emb.dim $R=1+\operatorname{dim}_{F} \Omega_{F / Q}$. Combining this with Corollary 5.2 completes the proof of the theorem.

Corollary 5.4. With the same assumptions as in the theorem, if also $F$ is separable over $Q$, then emb.dim $R=d$ and the ring $\widehat{A}_{P}$ is a regular local ring. 
Proof. Since $F$ is separable over $Q$, and $F$ is a finitely generated extension of $Q$, the dimension of $\Omega_{F / Q}$ is the transcendence degree of $F$ over $Q$ [5, Corollary 16.17(a), p. 403]. Thus by Theorem 5.3, emb.dim $R=1+\operatorname{tr} \cdot \operatorname{deg}_{Q} F$. Since $U$ is DVR, it is universally catenary (see for example [5, Corollary 18.10, p. 457]), and hence, since $A$ is essentially of finite type over $U$, the dimension formula holds for the extension $U \subseteq A$ [20, Theorem 15.5, p. 118]. In particular,

$$
\operatorname{dim}(A)+\operatorname{tr} \cdot \operatorname{deg}_{U /(\mathfrak{m} \cap U)} A / \mathfrak{m}=\operatorname{dim}(U)+\operatorname{tr} \cdot \operatorname{deg}_{Q} F .
$$

As noted in the proof of Theorem 5.1, $A=U+\mathfrak{m}$, so it follows that $A / \mathfrak{m} \cong$ $U /(\mathfrak{m} \cap U)$. Thus since $\operatorname{dim}(U)=1$, the dimension formula yields $d=\operatorname{dim}(A)=$ $1+$ tr. $\operatorname{deg}_{Q} F$, so that

$$
\text { emb.dim } R=1+\text { tr. } \operatorname{deg}_{Q} F=1+(d-1)=d .
$$

The corollary now follows from Theorem 5.3 .

Thus if the quotient field $Q$ of $U$ is perfect and $A$ is essentially of finite type over $U$, then $\widehat{A}_{P}$ is a regular local ring for every dimension 1 prime ideal $P$ of $\widehat{A}$ with $P \cap A=0$. This however is just a special case of the fact that a DVR with perfect quotient field is a $G$-ring; see [11, Remark 10.1].

\section{Analytic RAmification in POSITIVe CharaCteristic}

In this section we show that all bad stable domains in positive characteristic arise as in Section 4 from derivations. The reason for this is that in positive characteristic, all one-dimensional analytically ramified local Noetherian stable domains tightly dominated by a DVR occur within an immediate extension of DVRs. This is a consequence of a theorem of Bennett, which states that if $R$ is a one-dimensional local Noetherian domain of characteristic $p>0$, and there is a nilpotent prime ideal $P$ of $\widehat{R}$ such that $\widehat{R} / P$ is a DVR, then there is a DVR $U$ such that $U \subseteq R \subseteq \widehat{U}$ and $R^{q} \subseteq U$ for some $q=p^{e}$ [2, Theorem 1, p. 133]. This implies:

Lemma 6.1 (Bennett [2]). If the normalization of a Noetherian domain $A$ of positive characteristic is a DVR $V$ that tightly dominates $A$, then $A$ contains a $D V R U$ such that $U \subseteq V$ is an immediate extension.

We apply the lemma in Theorem 6.4 to show that all bad Noetherian stable domains in positive characteristic occur as the pullback of a derivation. The theorem depends on two lemmas that are valid in any characteristic.

Lemma 6.2. Let $V$ be a DVR with quotient field $F$, and let $Q$ be a subfield of $F$. Then $V$ is an immediate extension of the $D V R V \cap Q$ if and only if $F=V+Q$.

Proof. Let $U=V \cap Q$. If $V$ is an immediate extension of $U$, then $V / U$ is a torsionfree divisible $U$-module. Moreover, since $V$ is a DVR dominating $U$, it follows that $F=Q V$. Thus from the fact that $F=Q V$ and $V / U$ is divisible, we deduce that $F=Q V=V+Q$. Conversely, if $F=V+Q$, then $V / U=V /(Q \cap V) \cong$ $(V+Q) / Q=F / Q$, so that $V / U$ is a divisible torsion-free $U$-module, and hence $U \subseteq V$ is an immediate extension of DVRs.

Lemma 6.3. Let $V$ be a DVR with quotient field $F$, let $K$ be a torsion-free $V$ module such that $K / \mathfrak{M}_{V} K$ is a nonzero finitely generated $V$-module, and suppose 
$D: V \rightarrow F K$ is a derivation such that $F K$ is generated as an $F$-vector space by $D(V)$. If $V=\operatorname{Ker} D+c V$ for some $0 \neq c \in \mathfrak{M}_{V} \cap \operatorname{Ker} D$, then $R=D^{-1}(K)$ is a bad Noetherian stable domain.

Proof. Let $U=\operatorname{Ker} D$, and let $Q$ be the quotient field of $U$. We show first that $U \subseteq V$ is an immediate extension of DVRs. It suffices by Lemma 6.2 to verify that $U=V \cap Q$ and $F=V+Q$. Clearly, $U \subseteq V \cap Q$. Let $v \in V \cap Q$, and write $v=\frac{a}{b}$ where $a, b \in \operatorname{Ker} D$ with $b \neq 0$. Then since $D(b)=0$, we have $b D(v)=D(a)=0$, and since $K$ is torsion-free, $D(v)=0$. Thus $v \in \operatorname{Ker} D$, which shows that $V \cap Q=U$. Now to see that $F=V+Q$, observe that since $U$ contains a nonzero element in the maximal ideal of $V$, it suffices to show that $Q V \subseteq V+Q$. Let $0 \neq a \in \operatorname{Ker} D$ and $v \in V$. We claim $a^{-1} v \in Q+V$. Since $V$ is a DVR, there exists $k>0$ such that $c^{k} \in a V$, and hence from the assumption that $V=\operatorname{Ker} D+c V$, it follows that $V=\operatorname{Ker} D+a V$. Thus $v=b+a w$ for some $b \in \operatorname{Ker} D$ and $w \in V$, and hence $a^{-1} v=a^{-1} b+w \in Q+V$, which proves the claim that $U \subseteq V$ is immediate.

Now let $C=U \backslash\{0\}$. As noted above, $V_{C}=F$. Since by assumption $F K$ is generated as an $F$-vector space by $D(F)$, Proposition 3.5 implies that the mapping $R \rightarrow V \star K: r \mapsto(r, D(r))$ is an analytic isomorphism along $C$. As in the proof of Theorem 4.4 (1), this implies that the ring $\widehat{R}$ is isomorphic to $\widehat{V} \star \widehat{K}$. In particular, since $K / \mathfrak{M}_{V} K$ is a nonzero $V$-module, then $\widehat{K} \neq 0$, and hence there is a nonzero prime ideal $P$ of $\widehat{R}$ such that $P^{2}=0$ and $\widehat{R} / P$ is a DVR. Thus by (2.5), $R$ is a bad stable domain. Also, as in the proof of Theorem 4.4(2), since $K / \mathfrak{M}_{V} K$ is a finitely generated $V$-module, $R$ is a Noetherian domain.

Theorem 6.4. The following are equivalent for a quasilocal domain $(R, \mathfrak{m})$ of positive characteristic:

(1) $R$ is a bad Noetherian stable domain.

(2) The normalization $V$ of $R$ is a DVR and there exists a torsion-free $V$ module $K$ with $K / \mathfrak{M}_{V} K$ a nonzero finitely generated $V$-module, and a derivation $D: V \rightarrow F K$ such that $D(V)$ generates $F K$ as an F-vector space, $R=D^{-1}(K)$ and $V=\operatorname{Ker} D+\mathfrak{m} V$.

Proof. Suppose that $R$ is a bad Noetherian stable domain. Then by (2.4), $V$ is a DVR that tightly dominates $R$, and hence by Lemma 6.1 there exists a DVR $U \subseteq R$ such that $U \subseteq V$ is an immediate extension. By Theorem 4.1 $R=V \cap d_{F / Q}^{-1}(\Omega(R))$, where $Q$ and $F$ are the quotient fields of $U$ and $V$, respectively. By Theorem 4.4(2), $\Omega(R) / \mathfrak{M}_{V} \Omega(R)$ is a nonzero finitely generated $V$-module. Let $D$ be the restriction of $d_{F / Q}$ to $V$. By definition, $D(R)$ generates $\Omega(R)$ as a $V$-module, and hence $D(V)$ generates $F \Omega(R)=\Omega_{F / Q}$ as an $F$-vector space. Moreover, $R=D^{-1}(\Omega(R))$, and since $D$ is $U$-linear and $V / U$ is a divisible $U$-module, it follows that $V=\operatorname{Ker} D+c V$ for all $0 \neq c \in U$. Hence $V=\operatorname{Ker} D+\mathfrak{m} V$, and this verifies statement (2). The converse, that (2) implies (1), is given by Lemma 6.3 .

These ideas also lead to a characterization in Theorem 6.5 of the DVRs of positive characteristic which are the normalization of an analytically ramified local Noetherian domain. 
Theorem 6.5. The following are equivalent for a DVR $V$ having quotient field $F$ of positive characteristic $p$ :

(1) $V$ is the normalization of an analytically ramified local Noetherian domain.

(2) There exists a subfield $Q$ of $F$ such that $F=V+Q$ and $F \neq Q\left[F^{p}\right]$.

Proof. To see that (1) implies (2), let $R$ be an analytically ramified local Noetherian domain having normalization $V$. As discussed before Corollary 4.2, every onedimensional analytically ramified local Noetherian domain has a bad 2-generator overring. Thus there exists a bad Noetherian stable ring $S$ having normalization $V$, and by (2.4), $S$ is tightly dominated by $V$. Thus by Lemma 6.1, there exists a DVR $U \subseteq S$ such that $U \subseteq V$ is an immediate extension. Let $Q$ be the quotient field of $U$. Then by Corollary 4.2, $F \neq Q\left[F^{p}\right]$, and since $U \subseteq V$ is immediate, $V / U$ is a divisible $U$-module and $Q V=F$. Thus if $0 \neq u \in U$ and $v \in V$, writing $v=u^{\prime}+u w$ for some $u^{\prime} \in U$ and $w \in V$, we have $u^{-1} v=u^{-1} u^{\prime}+w \in Q+V$, which, since $F=Q V$, proves that $F=V+Q$. The converse, that (2) implies (1), follows from Corollary 4.2 and Lemma 6.2

\section{PRescribed NORMAlization}

The DVRs of positive characteristic that are the normalization of an analytically ramified local Noetherian domain were characterized in Theorem 6.5, In this section we describe additional cases where a given DVR is the normalization of an analytically ramified local ring. The first case involves equicharacteristic DVRs with a "large" quotient field.

Theorem 7.1. Let $V$ be an equicharacteristic DVR with quotient field $F$ having a countable subfield $k$ contained in $V$ such that $F$ is separably generated and of infinite transcendence degree over $k$. Then for every $d>1, V$ is the normalization of a bad Noetherian stable ring of embedding dimension $d$. Moreover, $V$ is the normalization of a bad stable domain that is not Noetherian.

Proof. In [24, Lemmas 3.1 and 3.2] it is shown that since $F / k$ is a separably generated field extension of infinite transcendence degree, where $k$ has at most countably many elements and $V$ is a $k$-subalgebra of $F$ having quotient field $F$, then for any $t \in V$ there exists a ring $U$ such that $k[t] \subseteq U \subseteq V, V / U$ is a torsion-free divisible $U$-module, and, with $Q$ the quotient field of $U, \operatorname{dim}_{F} \Omega_{F / Q}$ is infinite. Thus, choosing $t \in V$ such that $t V$ is the maximal ideal of $V$, there exists a subring $U$ of $V$ such that $V / U$ is a torsion-free divisible module and $t \in U$. The fact that $V / U$ is torsion-free implies that $U=Q \cap V$. Also since $V / U$ is divisible and $F=V Q$, then $F=V+Q$, so that by Lemma 6.2, $U \subseteq V$ is an immediate extension of DVRs with $\operatorname{dim}_{F} \Omega_{F / Q}$ infinite. Theorems 4.1 and 4.4 now complete the proof.

Since field extensions in characteristic 0 are separable, we deduce

Corollary 7.2. If $V$ is a DVR of equicharacteristic 0 that has infinite transcendence degree over its prime subfield, then for each $d>1, V$ is the normalization of a bad Noetherian stable domain of embedding dimension $d$.

In particular, every uncountable DVR of equicharacteristic 0 is the normalization of a bad Noetherian stable domain.

Next we consider the case of algebraic function fields $F / k$, and we characterize the DVRs in $F / k$ that are the normalization of an analytically ramified local 
Noetherian $k$-algebra. The emphasis in the theorem is thus on staying inside the category of $k$-algebras. By a DVR in $F / k$ we mean a DVR that is a $k$-algebra having quotient field $F$. A divisorial valuation ring in $F / k$ is a DVR $V$ in $F / k$ such that tr. $\operatorname{deg}_{k} V / \mathfrak{M}_{V}=$ tr. $\operatorname{deg}_{k} F-1$.

Theorem 7.3. Let $F / k$ be a finitely generated field extension, and let $V$ be a DVR in $F / k$ such that $V / \mathfrak{M}_{V}$ is a finitely generated extension of $k$. Then the following statements are equivalent:

(1) $V$ is the normalization of an analytically ramified local Noetherian domain containing $k$.

(2) $V$ is the normalization of a bad stable ring containing $k$ and having embedding dimension $d=\operatorname{tr} \cdot \operatorname{deg}_{k} F-\operatorname{tr} \cdot \operatorname{deg}_{k} V / \mathfrak{M}_{V}$.

(3) $V$ is not a divisorial valuation ring in $F / k$.

Proof. That (2) implies (1) is clear. To see that (1) implies (3), let $R$ be an analytically ramified local ring having normalization $V$, and suppose by way of contradiction that $V$ is a divisorial valuation. Then, as noted in the proof of Theorem 6.5. we may assume without loss of generality that $R$ is a bad stable ring having normalization $V$; as such, since by (2.4), $V / R$ is a divisible $R$-module, $R$ has the same residue field as $V$. Thus with $n=\operatorname{tr} \cdot \operatorname{deg}_{k} F$ and $M$ the maximal ideal of $R$, there exist units $x_{1}, \ldots, x_{n-1} \in R$ such that the images in $R / M$ of these elements are algebraically independent over $k$. Therefore, if $0 \neq f \in k\left[X_{1}, \ldots, X_{n-1}\right]$, then $f\left(x_{1}, \ldots, x_{n-1}\right) \notin M$, and this implies that $k\left(x_{1}, \ldots, x_{n-1}\right) \subseteq R$. Since $R$ has quotient field $F$, there is a finitely generated $k\left(x_{1}, \ldots, x_{n-1}\right)$-subalgebra $A$ of $R$ with quotient field $F$. Since tr. $\operatorname{deg}_{k} F=n$, it follows that $A$ is a one-dimensional Noetherian domain, and as a finitely generated algebra over a field, $A$, and hence every overring of $A$, has finite normalization. (Indeed, if $B$ is an overring of $A$ and $\bar{A}$ is the normalization of $A$, then since $\bar{A}$ is a Dedekind domain, it follows that $B \bar{A}$ is the normalization of $B$. Thus since $A$ has finite normalization, then so does $B$.) But since $R$ is analytically ramified, $R$ does not have finite normalization, and this contradiction implies that $V$ is not a divisorial valuation ring.

Finally, to see that (3) implies (2), let $r=\operatorname{tr} \cdot \operatorname{deg}_{k} V / \mathfrak{M}_{V}$. Then as above, there exist algebraically $k$-independent elements $x_{1}, \ldots, x_{r}$ of $V$ such that $k^{\prime}:=$ $k\left(x_{1}, \ldots, x_{r}\right) \subseteq V$. Let $A$ be a finitely generated $k^{\prime}$-subalgebra of $V$ with quotient field $F$. Then since $V / \mathfrak{M}_{V}$ is algebraic over $k^{\prime}, \mathfrak{m}:=\mathfrak{M}_{V} \cap A$ is a maximal ideal of $A$. Since also $V / \mathfrak{M}_{V}$ is finite over $k^{\prime}$, we may enlarge $A$ by adjoining finitely many elements of $V$ to obtain that $V$ tightly dominates $B:=A_{\mathfrak{m}}$. Now $B$ has Krull dimension tr. $\operatorname{deg}_{k} F-r=d>1$ (here we are using the assumption that $r<d-1$ ), so as discussed before Corollary [5.2, there is a bad stable ring $S$ having normalization $V$ and embedding dimension 1 more than the embedding dimension of $\widehat{B}_{P}$, where $P$ is a certain dimension one prime ideal of $\widehat{B}$ with $P \cap B=0$. Since $B$ is excellent, the ring $\widehat{B}_{P}$ is a regular local ring, and hence its embedding dimension is $d-1$. Therefore, $S$ has embedding dimension $d$.

We next consider two cases, one in which $V$ is a DVR that contains a coefficient field and the other a special instance of mixed characteristic. In the former case we can describe some of the bad stable subrings of $V$, while in the latter we can describe all the bad stable subrings of $V$ which share their quotient field with $V$ and for which the relevant prime integer $p$ of $V$ is not a unit. 
Proposition 7.4. Let $V$ be a DVR with residue field $k$ and quotient field $F$, and let $t \in V$ be such that $t V$ is the maximal ideal of $V$. Then the following statements hold for $V$ :

(1) Suppose $V=k+t V$. If $\Omega_{F / k(t)}=0$, then no ring containing $k[t]$ and having normalization $V$ is an analytically ramified local Noetherian domain. Otherwise, if $\Omega_{F / k(t)} \neq 0$, then there is a one-to-one correspondence between the proper full $V$-submodules of $\Omega_{F / k(t)}$ and the bad stable rings containing $k[t]_{(t)}$ and having normalization $V$.

(2) Suppose $V$ has characteristic $0, k$ has exactly $p$ elements ( $p$ a prime), and $p V$ is the maximal ideal of $V$. If $F / \mathbb{Q}$ is algebraic, where $\mathbb{Q}$ is the field of rational numbers, then no proper subring of $V$ having quotient field $F$ and normalization $V$ is an analytically ramified local Noetherian domain. Otherwise, if $F / \mathbb{Q}$ is not algebraic, then there is a one-to-one correspondence between the full $V$-submodules of $\Omega_{F / \mathbb{Q}}$ and the quasilocal one-dimensional stable rings $R$ birationally dominated by $V$ such that $p R \neq R$.

(3) For any such proper stable subring $R$ of $V$ arising as in (1) or (2), $R$ is a bad stable domain with normalization $V$. Moreover, $R$ can be chosen to be Noetherian of embedding dimension $n+1$, as long as $n$ is selected in (1) such that $1 \leq n \leq \operatorname{dim}_{F} \Omega_{F / k(t)}$, and in (2) such that $1 \leq n \leq \operatorname{dim}_{F} \Omega_{F / \mathbb{Q}}$. If either of these vector space dimensions is infinite, then in the corresponding case, $R$ can be chosen to be a non-Noetherian bad stable domain.

Proof. (1) With $U:=k[t]_{(t)}$, the extension $U \subseteq V$ is immediate, so by Theorem 4.1 there is a one-to-one correspondence between the nonzero full $V$-submodules of $\Omega_{F / k(t)}$ and the stable rings $R$ occurring between $U$ and $V$ and having normalization $V$. Suppose that $R$ is an analytically ramified local Noetherian domain having normalization $V$ and containing $U$. Then $R$ has dimension 1, and by Theorem 5.1 there exists a bad Noetherian stable ring between $R$ and $V$, in which case by the correspondence, $\Omega_{F / k(t)} \neq 0$.

(2) We view $U:=\mathbb{Z}_{(p)}$ (the ring of integers localized at the prime ideal $(p)$ ) as a subring of $V$. Then $U=V \cap \mathbb{Q}$, and since the residue field of $V$ has $p$ elements and the maximal ideal of $V$ is $p V$, we have $V=U+p V$. Hence $U \subseteq V$ is an immediate extension. Statement (2) now follows as in (1) from Theorems 4.1 and 5.1 . (Here we are using implicitly that $F / \mathbb{Q}$ is algebraic if and only if $\Omega_{F / \mathbb{Q}}=0$; see (2.2).)

(3) Let $Q$ denote the quotient field of $U$, where $U$ is as in (1) or (2), depending on which case we wish to consider. Let $n$ be such that $n \leq \operatorname{dim}_{F} \Omega_{F / Q}$, where we admit the possibility that $n$ is infinite. Let $K$ be a full $V$-submodule of $\Omega_{F / Q}$ such that $K$ is the direct sum of a rank $n$ free $V$-submodule of $\Omega_{F / Q}$ and a divisible $V$-submodule. With $R:=V \cap d_{F / Q}^{-1}(K)$, we have by Theorem 4.1 that $R$ is a bad stable domain. Moreover, by Theorem 4.4, $R$ is Noetherian of embedding dimension $n+1$ if and only if $n$ is finite. Also by Theorem 4.4, $n \leq \operatorname{dim}_{F} \Omega_{F / Q}$. This proves (3).

In Theorem 7.6 we apply the proposition to determine when certain complete DVRs are the normalization of an analytically ramified local Noetherian domain. The theorem depends on the next lemma, where we calculate the dimension of $\Omega_{k((X)) / k(X)}$ in two cases. In the first case in which $k$ is perfect of characteristic $p \neq 0$ (i.e., $k=k^{p}$ ), we see that the module of Kähler differentials is trivial, while in a case at the other extreme, where not only is $k \neq k^{p}$ but $\left[k: k^{p}\right]$ is uncountable, 
then this module has infinite dimension as a $k((X))$-vector space. Statement $(2)$ of the lemma is mentioned without proof in [6, Remark 3.7(i)]. We supply a proof here for the sake of completeness.

Lemma 7.5. Let $k$ be a field of positive characteristic $p$, and let $X$ be an indeterminate for $k$.

(1) If $k$ is perfect, then $\Omega_{k((X)) / k(X)}=0$.

(2) If $\left[k: k^{p}\right]$ is uncountable, then $\Omega_{k((X)) / k(X)}$ has infinite dimension as a $k((X))$-vector space.

Proof. (1) Let $F=k((X))$. Then to show that $\Omega_{F / k(X)}=0$, it is by (2.2) sufficient (and necessary) to show that $F=k(X)\left[F^{p}\right]$. Moreover, since $k(X)\left[F^{p}\right]$ is a field and $F$ is the quotient field of $k[[X]]$, we need only show that $k[[X]] \subseteq k(X)\left[F^{p}\right]$. To this end, let $z \in k[[X]]$, and write $z=\sum_{i=0}^{\infty} \alpha_{i} X^{i}$, where $\alpha_{i} \in k$. For each $j=0,1, \ldots, p-1$, define

$$
z_{j}=\sum_{i=0}^{\infty} \alpha_{i p+j} X^{i p+j}
$$

so that $z=z_{0}+z_{1}+\cdots+z_{p-1}$. Then since $k$ is perfect of characteristic $p$,

$$
z_{j}=\sum_{i=0}^{\infty} \alpha_{i p+j} X^{i p+j}=X^{j} \sum_{i=0}^{\infty} \alpha_{i p+j} X^{i p}=X^{j}\left(\sum_{i=0}^{\infty}\left(\alpha_{i p+j}\right)^{1 / p} X^{i}\right)^{p} .
$$

Therefore, $z_{j} \in k(X)\left[F^{p}\right]$, so that $z=z_{0}+\cdots+z_{p-1} \in k(X)\left[F^{p}\right]$, proving that $F=k(X)\left[F^{p}\right]$.

(2) Let $F=k\left[k^{p}\left(\left(X^{p}\right)\right)\right]$. We first claim that $[k((X)): F]$ is infinite. Suppose the contrary. Then there exist $z_{1}, \ldots, z_{n} \in k((X))$ such that

$$
k((X))=F z_{1}+\cdots+F z_{n} .
$$

Now there exists $m \in \mathbb{Z}$ such that each $z_{j}$ is of the form $z_{j}=\sum_{i>m} \delta_{i} X^{i}$, where $\delta_{i} \in k$. Let $L$ be the subfield of $k$ generated by $k^{p}$ and all the coefficients $\delta_{i}$ of all the $z_{j}$. Then $\left[L: k^{p}\right]$ is countable, so since $\left[k: k^{p}\right]$ is uncountable, the $L$ vector space $k$ has infinite dimension. With this in mind, let $\alpha_{0}, \alpha_{1}, \alpha_{2}, \ldots$ be elements of $k$ that are linearly independent over $L$, and define $w=\sum_{i \geq 0} \alpha_{i} X^{i}$. Then since $w \in k((X))=F z_{1}+\cdots+F z_{n}$, there exist $f_{1}, \ldots, f_{n} \in F$ such that $w=f_{1} z_{1}+\cdots+f_{n} z_{n}$. Now there exists $t>0$ such that for each $j=1,2, \ldots, n$, we may write

$$
f_{j}=\beta_{j 1} \sum_{i} \gamma_{j 1 i}^{p} X^{i p}+\cdots+\beta_{j t} \sum_{i} \gamma_{j t i}^{p} X^{i p}
$$

where $\beta_{j \ell}, \gamma_{j m i} \in k$, and $i$ is allowed to range over some set of integers containing a lower bound. Then

$$
f_{j}=\sum_{i}\left(\beta_{j 1} \gamma_{j 1 i}^{p}+\cdots+\beta_{j t} \gamma_{j t i}^{p}\right) X^{i p},
$$

and so the coefficients of $f_{j} z_{j}$ are in the $L$-vector space $L \beta_{j 1}+\cdots+L \beta_{j t}$. Hence the coefficients of $w=f_{1} z_{1}+\cdots+f_{n} z_{n}$ are in the finite-dimensional $L$-subspace of $k$ generated by $\left\{\beta_{j \ell}: 1 \leq j \leq n, 1 \leq \ell \leq t\right\}$. But then $\alpha_{0}, \alpha_{1}, \ldots$ are in this finitedimensional $L$-subspace of $F$, which contradicts the choice of the $\alpha_{i}$ as elements of $F$ which are linearly independent over $L$. This proves that $[k((X)): F]$ is infinite. 
Now to show that $\Omega_{k((X)) / k(X)}$ has infinite dimension as a $k((X))$-vector space, it suffices to show that $k((X))$ has an infinite $p$-basis over $k(X)$; see [20, Theorem 26.5 , p. 202]. Thus, since $k((X))^{p}=k^{p}\left(\left(X^{p}\right)\right)$, it is enough to prove that

$$
[k((X)): k(X)[F]]=\infty .
$$

Observe that

$$
[k((X)): F]=[k((X)): k(X)[F]] \cdot[k(X)[F]: F],
$$

and since $[k((X)): F]$ is infinite and $[k(X)[F]: F]$ is finite (note that $X$ is algebraic over $F$ ), we conclude that $[k((X)): k(X)[F]]$ is infinite, and this proves $(2)$.

Theorem 7.6. Let $V$ be a complete DVR with residue field $k$.

(1) If $V$ has characteristic $p \neq 0$ and $k$ is perfect, then there does not exist an analytically ramified local Noetherian domain containing $k$ whose normalization is $V$.

(2) If either (a) $V=\widehat{\mathbb{Z}}_{p}$, (b) $V$ and $k$ have characteristic 0 , or (c) $V$ has characteristic $p \neq 0$ and $\left[k: k^{p}\right]$ is uncountable, then for every $d>1$ there exists a bad Noetherian stable domain of embedding dimension d whose normalization is $V$. There also exists a non-Noetherian bad stable domain whose normalization is $V$.

Proof. (1) By the Cohen structure theorem for complete local rings, we may assume that $k \subseteq V$, where $k$ is as in (1); see 3]. Suppose there exists an analytically ramified local Noetherian domain that contains $k$ and whose normalization is $V$. Then, as noted before Theorem 6.5, a theorem of Matlis shows there exists a bad Noetherian stable domain $R$ that contains $k$ and whose normalization is $V$. Since $V / R$ is a divisible $R$-module, the maximal ideal of $V$ is extended from the maximal ideal of $R$, and hence since $V$ is a DVR, there exists $t \in R$ such that $t V$ is the maximal ideal of $V$. Then $k[t]_{(t)} \subseteq R$, and by the structure theorem, $V=k[[t]]$. But by Lemma 7.5, $\Omega_{k((t)) / k(t)}=0$, so by Proposition 7.4(1), no such ring $R$ can exist.

(2) If $V=\widehat{\mathbb{Z}}_{p}$, then since $\widehat{\mathbb{Q}}_{p}$ has infinite transcendence degree over the characteristic 0 field $\mathbb{Q}$, the $\widehat{\mathbb{Q}}_{p}$-vector space $\Omega_{\widehat{\mathbb{Q}}_{p} / \mathbb{Q}}$ has infinite dimension [5, Corollary A1.5(a), p. 567], and hence the claim follows from Proposition 7.4(2) and (3). The case where $V$ and $k$ have characteristic 0 is decided by Corollary 7.2 Finally, if $V$ has characteristic $p \neq 0$ (so that also $k$ has characteristic $p$ ) and $\left[k: k^{p}\right]$ is uncountable, then $V=k[[t]]$ for some $t \in V$, and by Lemma $\left[7.5(2), \Omega_{k((t)) / k(t)}\right.$ has infinite dimension as a vector space over $k((t))$. In this case we also apply Proposition 7.4 to prove the claim.

An immediate consequence of the theorem is that there exist non-Noetherian stable domains of Krull dimension 1:

Corollary 7.7. Let $k$ be a field either of characteristic 0 or of characteristic $p \neq 0$ such that $\left[k: k^{p}\right]$ is uncountable, and let $X$ be an indeterminate for $k$. Then there exists a non-Noetherian bad stable domain $R$ whose normalization is $k[[X]]$.

\section{LOCAL RINGS WITH NORMALIZATION A COMPLETE DVR}

In this section we examine more closely the case in which an analytically ramified local Noetherian domain has normalization a complete DVR. In particular, we use Matlis' theory of $Q$-rings to characterize in Theorem 8.4 the bad 2-generator 
domains whose normalization is a complete DVR, and with this we recover in Corollary 8.5 a characterization due to Matlis of the analytically ramified local Noetherian domains having normalization a complete DVR.

In the article [19], Matlis introduced the notion of a $Q$-ring to study the domains $R$ with quotient field $Q$ which have an $R$-module $L$ such that $\operatorname{Hom}_{R}(Q, L)=0$ and $\operatorname{Ext}_{R}^{1}(Q, L) \cong Q$. The motivation for studying such rings was a question from singular cohomology (ultimately answered in the negative) as to whether the ring of integers possessed such a module. Matlis proved in Theorem 2.1 of [19] that the existence of such an $R$-module was equivalent to $R$ being what he termed a " $Q$-ring", which we define momentarily. The $Q$-rings fall into three classes, depending on the number of proper nonzero $h$-divisible $R$-submodules of $Q / R$, where an $R$-module $L$ is $h$-divisible if $L$ is a homomorphic image of a divisible torsion-free $R$-module. If $R$ is a $Q$-ring, then $Q / R$ has either 0,1 or 2 proper nonzero $h$-divisible $R$-submodules [19, Theorem 3.4].

An integral domain $R$ with quotient field $Q$ is a $Q$-ring if $\operatorname{Ext}_{R}^{1}(Q, R) \cong Q$. The domain $R$ is a $Q_{0^{-}}, Q_{1^{-}}$, or $Q_{2^{-}}$ring depending on whether $Q / R$ contains 0,1 or 2 proper nonzero $h$-divisible $R$-submodules. In the case where $F$ denotes the quotient field of $R$, we still use the term $Q$-ring (as opposed to, say, $F$-ring) to describe the situation in which $\operatorname{Ext}_{R}^{1}(F, R) \cong F$. Recall that the rank of a torsion-free module $L$ over a domain $R$ with quotient field $F$ is the dimension of the $F$-vector space $F \otimes_{R} L$. The following lemma uses the notion of completion in the ideal topology, which was discussed in (2.5): If $R$ is a domain, then $\widetilde{R}=\lim R / r R$, where $r$ ranges over the nonzero elements of $R$. The rank of the completion of $R$ determines whether $R$ is a $Q$-ring:

Lemma 8.1 (Matlis [19, Theorem 2.2]). A domain $R$ is a $Q$-ring if and only if the rank of the torsion-free $R$-module $\widetilde{R}$ is 2 , where the completion is with respect to the ideal topology on $R$.

We now consider $Q_{1}$-domains whose normalization is a DVR.

Lemma 8.2 (Matlis [19, Theorem 4.3]). Let $R$ be a $Q_{1}$-domain with quotient field $F$, and let $B / R$ be the unique proper nonzero $h$-divisible $R$-submodule of $F / R$. Then $B$ is a ring and there is a unique minimal prime ideal $P$ of $\widetilde{R}$, where $\widetilde{R}$ is the completion of $R$ in the ideal topology, such that $\widetilde{R} / P \cong B ; P \cong \operatorname{Hom}_{R}(F / R, B / R)$; and $P^{2}=0$.

The assertion that $P^{2}=0$ is not in the statement of Matlis' theorem, but it can be found in the proof. The next lemma follows from results in [25] and can be viewed as another characterization of bad 2-generator domains.

Lemma 8.3. Let $R$ be a bad stable domain with normalization $V$ and quotient field $F$. Then every $R$-submodule of $V / R$ admits a unique $V$-module structure extending the $R$-module structure, and the following statements are equivalent:

(1) $V / R$ is an indecomposable $R$-module.

(2) $V / R$ has no proper nonzero divisible $R$-submodules.

(3) $V / R$ is the unique proper nonzero divisible $R$-submodule of $F / R$.

(4) $R$ is a bad 2-generator ring.

Proof. Since $V / R$ is a torsion divisible $R$-module and $R \subseteq V$ is a quadratic extension, we may apply Lemma 3.1(4) to obtain that $V / R$ is a $V$-module, and from 
this it follows that this $V$-module structure induces a $V$-module structure on every $R$-submodule of $V / R$; see [25. Lemma 3.1].

$(1) \Rightarrow(2)$ Suppose that $B / R$ is a proper nonzero divisible $R$-submodule of $V / R$. As above, $B / R$ is a $V$-submodule of $V / R$, and since $R$ and $V$ share the same quotient field, it follows that $B / R$ is a divisible $V$-submodule of $V / R$. Then since $V$ is a DVR, $B / R$ is an injective $V$-module, and hence is a summand of $V / R$. This shows that if $V / R$ is indecomposable, then there are no nonzero proper divisible $V$-submodules of $V / R$.

$(2) \Rightarrow(3)$ Suppose that $B$ is an $R$-submodule of $F$ containing $R$ such that $B / R$ is a proper nonzero divisible $R$-submodule of $F / R$. A straightforward argument shows that the divisibility of this module implies that $B$ is a ring. But since the normalization of $R$ is $V$ and $R \subseteq B$, it follows that $V$ is a subring of the normalization of $B$. Yet $V$ is a DVR, so this forces $V$ to be the normalization of $B$, and hence $B \subseteq V$. Thus by $(2), B / R=V / R$.

$(3) \Rightarrow(1)$ Suppose that $V / R=\left(B_{1} / R\right) \oplus\left(B_{2} / R\right)$ for some $R$-submodules $B_{1}$ and $B_{2}$ of $V$ containing $R$. Then as an image of $V / R$, each of $B_{1} / R$ and $B_{2} / R$ is a divisible $R$-module. Thus by (3), $B_{1}=V$ or $B_{2}=V$, and hence $V / R$ is an indecomposable $R$-module.

$(1) \Rightarrow(4)$ Assume (1). Then by (2.4), $V / R$ is isomorphic to $F / V$ as $V$-modules. It follows that the $V$-submodules of $V / R$ form a chain under inclusion. But, as noted above, every $R$-submodule of $V / R$ is also a $V$-submodule, so the set of $R$ submodules of $V / R$ forms a chain. Let $M$ denote the maximal ideal of $R$, and let $R_{1}=\{q \in F: q M \subseteq M\}$. Then since $M$ is a stable ideal, $M=m R_{1}$ for some $m \in M$. If $R_{1}=R$, then $M$ is a principal ideal of $R$ and $R=V$, a contradiction to the assumption that $R$ is a bad stable domain. Thus $R_{1} / R$ is a nonzero $R / M$ vector space, and since $R_{1} / R \subseteq V / R$ and the $R$-submodules of $V / R$ form a chain, it must be that $R_{1} / R$ has dimension 1 as a vector space. Therefore, there exists $x \in R_{1}$ such that $R_{1}=x R+R$, and consequently, $M=m R_{1}=m x R+m R$. Thus $R$ is a stable domain with a maximal ideal that can be generated by 2 elements. Therefore, since the maximal ideal has reduction number $\leq 1$, the multiplicity of $R$ is at most 2. Consequently, every ideal of $R$ can be generated by 2 elements [28, Theorem 1.1, p. 49].

$(4) \Rightarrow(1)$ Assume (4). Since every ideal of $R$ can be generated by 2 elements, the $R$-submodules of $V / R$ form a chain under inclusion (see for example [23, Lemma $3.5]$ ), and hence $V / R$ is indecomposable.

With the lemma, we characterize bad 2-generator domains with normalization a complete DVR.

Theorem 8.4. A ring $R$ is a bad 2-generator domain whose normalization $V$ is a complete $D V R$ if and only if $R$ is a one-dimensional quasilocal $Q_{1}$-ring tightly dominated by $V$.

Proof. Suppose that $R$ is a bad 2-generator domain with $V$ a complete DVR. Then by Lemma 8.3, $V / R$ is the unique proper nonzero divisible $R$-submodule of $F / R$ (where $F=$ quotient field of $R$ ) and it follows that $V$ tightly dominates $R$. Also, by (2.5), there exists a prime ideal $P$ of $\widetilde{R}$ such that $P^{2}=0$ and $\widetilde{R} / P \cong V$. (Here the completion is with respect to the ideal topology.) A theorem of Matlis shows that in this case, $P \cong \operatorname{Hom}_{R}(F / V, V / R)$ [18, Theorem 2.6]. But since $R$ is a bad 2-generator domain, $V / R \cong F / V(2.4)$, so that $P \cong \operatorname{Hom}_{R}(F / V, F / V)$. 
As a ring, $\operatorname{Hom}_{R}(F / V, F / V)$ is isomorphic to $\widehat{V}$ [18, Theorem 2.2], so we then see that $P \cong \widetilde{V} \cong V$, since $V$ is a complete DVR. Thus since $P$ is isomorphic to the $R$-submodule $V$ of $F, P$ has rank 1 as a torsion-free $R$-module, as does $\widetilde{R} / P \cong V$, so necessarily $\widetilde{R}$ has rank 2 as a torsion-free $R$-module. By Lemma 8.1 $R$ is a $Q$-ring, and hence since $V / R$ is the unique proper nonzero divisible $R$-submodule of $F / R, R$ is a $Q_{1}$-ring. (Recall here that divisible $=h$-divisible for quasilocal domains of Krull dimension 1, or, more generally, for Matlis domains [7, Theorem VII.2.8, p. 253].)

Conversely, suppose that $R$ is a $Q_{1}$-ring with $V / R$ a divisible $R$-module. Then by Lemma 8.2 there exists a prime ideal $P$ of $\widetilde{R}$ such that $P^{2}=0$ and $\widetilde{R} / P \cong V$. Therefore, by (2.5), $R$ is a bad stable domain. Moreover, since $R$ is a $Q_{1}$-ring and $V / R$ is $h$-divisible, it follows that $V / R$ contains no proper nonzero divisible $R$-submodules. Therefore, by Lemma 8.3 , $R$ is a bad 2 -generator ring.

From the theorem, we recover Matlis' characterization of Noetherian domains with normalization a complete DVR [19, Theorem 6.7]:

Corollary 8.5 (Matlis). Let $R$ be a one-dimensional analytically ramified local Noetherian domain. Then the normalization $V$ of $R$ is a complete DVR if and only if there exists a $Q_{1}$-ring $T$ with $R \subseteq T \subseteq V$. Moreover, the $Q_{1}$-ring $T$ can be chosen to be a bad 2-generator ring.

Proof. Suppose $V$ is a complete DVR. As discussed before Corollary 4.2 , there exists a bad 2-generator ring $T$ that birationally dominates $R$. Since necessarily $T \subseteq V$ and hence $T$ has a local normalization, it must be that $V$ is the normalization of $T$. Therefore, by Theorem 8.4 $T$ is a $Q_{1}$-ring.

Conversely, suppose that $R \subseteq T \subseteq V$ with $T$ a $Q_{1}$-ring. Let $B / T$ be the unique proper nonzero $h$-divisible $T$-submodule of $F / T$. Then by Lemma 8.2, $\widehat{R} / P \cong B$ for some prime ideal $P$ of $\widehat{R}$. Therefore, $B$ is a complete local Noetherian domain, and as such its normalization is a complete DVR (see for example [18, Theorem 10.4 , p. 93]). Since $B \subseteq V$, this forces the normalization of $B$ to be $V$, and hence $V$ is a complete DVR.

In [19, Matlis constructed a $Q_{1}$-ring in characteristic 2 and asked whether there exist $Q_{1}$-rings in any other characteristic. Using the results in this section, we find many other such examples in all characteristics:

Corollary 8.6. Let $V$ be a complete DVR with residue field $k$, and suppose that either:

(a) $k$ has characteristic 0 ,

(b) $V$ and $k$ have characteristic $p \neq 0$ and $\left[k: k^{p}\right]$ is uncountable, or

(c) $V=\widehat{\mathbb{Z}}_{p}$.

Then there exists a $Q_{1}$-ring $R$ such that $R$ is a bad 2-generator ring and the normalization of $R$ is $V$.

Proof. In all cases, Theorem 7.6 implies that there exists a bad Noetherian stable domain whose normalization is $V$. An application of Corollary 8.5 now completes the proof.

\section{ACKNOWLEDGMENT}

The author thanks the referee for helpful comments that improved the paper. 


\section{REFERENCES}

[1] Y. Akizuki, Einige Bemerkungen über primäre Integritätsbereiche mit Teilerkettensatz, Proc. Phys.-Math. Soc. Japan 17 (1935), 327-336.

[2] Bruce Bennett, On the structure of non-excellent curve singularities in characteristic $p$, Inst. Hautes Études Sci. Publ. Math. 42 (1973), 129-170. MR0318144 (47 \#6693)

[3] I. S. Cohen, On the structure and ideal theory of complete local rings, Trans. Amer. Math. Soc. 59 (1946), 54-106. MR0016094 (7,509h)

[4] Ada Maria de Souza Doering and Yves Lequain, Maximally differential prime ideals, J. Algebra 101 (1986), no. 2, 403-417, DOI 10.1016/0021-8693(86)90201-2. MR847167 (87j:13004)

[5] David Eisenbud, Commutative algebra, Graduate Texts in Mathematics, vol. 150, SpringerVerlag, New York, 1995. With a view toward algebraic geometry. MR1322960 (97a:13001)

[6] Daniel Ferrand and Michel Raynaud, Fibres formelles d'un anneau local noethérien (French), Ann. Sci. École Norm. Sup. (4) 3 (1970), 295-311. MR0272779 (42 \#7660)

[7] László Fuchs and Luigi Salce, Modules over non-Noetherian domains, Mathematical Surveys and Monographs, vol. 84, American Mathematical Society, Providence, RI, 2001. MR.1794715 (2001i:13002)

[8] K. R. Goodearl and T. H. Lenagan, Constructing bad Noetherian local domains using derivations, J. Algebra 123 (1989), no. 2, 478-495, DOI 10.1016/0021-8693(89)90057-4. MR1000498 (90g:13011)

[9] William Heinzer, David Lantz, and Kishor Shah, The Ratliff-Rush ideals in a Noetherian ring, Comm. Algebra 20 (1992), no. 2, 591-622, DOI 10.1080/00927879208824359. MR.1146317 (93c:13002)

[10] William Heinzer, Christel Rotthaus, and Judith D. Sally, Formal fibers and birational extensions, Nagoya Math. J. 131 (1993), 1-38. MR.1238631 (95a:13008)

[11] F. J. Herrera Govantes, M. A. Olalla Acosta, M. Spivakovsky, and B. Teissier, Extending a valuation centred in a local domain to the formal completion, Proc. Lond. Math. Soc. (3) 105 (2012), no. 3, 571-621, DOI 10.1112/plms/pds002. MR2974200

[12] Shihoko Ishii, Jet schemes, arc spaces and the Nash problem (English, with English and French summaries), C. R. Math. Acad. Sci. Soc. R. Can. 29 (2007), no. 1, 1-21. MR2354631 (2008j:14019)

[13] W. Krull, Dimensionstheorie in Stellenringen, J. Reine Angew. Math. 179 (1938), 204-226.

[14] Ernst Kunz, Kähler differentials, Advanced Lectures in Mathematics, Friedr. Vieweg \& Sohn, Braunschweig, 1986. MR 864975 (88e:14025)

[15] Christer Lech, A method for constructing bad Noetherian local rings, Algebra, algebraic topology and their interactions (Stockholm, 1983), Lecture Notes in Math., vol. 1183, Springer, Berlin, 1986, pp. 241-247, DOI 10.1007/BFb0075463. MR846452 (87m:13010a)

[16] Yves Lequain, Differential simplicity and complete integral closure, Pacific J. Math. 36 (1971), 741-751. MR0284422(44 \#1649)

[17] Joseph Lipman, Stable ideals and Arf rings, Amer. J. Math. 93 (1971), 649-685. MR0282969 (44 \#203)

[18] Eben Matlis, 1-dimensional Cohen-Macaulay rings, Lecture Notes in Mathematics, Vol. 327, Springer-Verlag, Berlin, 1973. MR.0357391 (50 \#9859)

[19] Eben Matlis, The theory of Q-rings, Trans. Amer. Math. Soc. 187 (1974), 147-181. MR0340241 (49 \#4996)

[20] Hideyuki Matsumura, Commutative ring theory, Cambridge Studies in Advanced Mathematics, vol. 8, Cambridge University Press, Cambridge, 1986. Translated from the Japanese by M. Reid. MR 879273 (88h:13001)

[21] Masayoshi Nagata, Local rings, Interscience Tracts in Pure and Applied Mathematics, No. 13, Interscience Publishers, a division of John Wiley \& Sons, New York-London, 1962. MR0155856(27 \#5790)

[22] Bruce Olberding, On the structure of stable domains, Comm. Algebra 30 (2002), no. 2, 877895, DOI 10.1081/AGB-120013188. MR.1883031 (2002m:13022)

[23] Bruce Olberding, Stability, duality, 2-generated ideals and a canonical decomposition of modules, Rend. Sem. Mat. Univ. Padova 106 (2001), 261-290. MR.1876223 (2002k:13042)

[24] Bruce Olberding, A counterpart to Nagata idealization, J. Algebra 365 (2012), 199-221, DOI 10.1016/j.jalgebra.2012.05.002. MR 2928459 
[25] B. Olberding, Generic formal fibers and analytically ramified stable rings, Nagoya Math. J., to appear.

[26] Bruce Olberding, Noetherian rings without finite normalization, Progress in commutative algebra 2, Walter de Gruyter, Berlin, 2012, pp. 171-203. MR2932595

[27] Ana J. Reguera, Towards the singular locus of the space of arcs, Amer. J. Math. 131 (2009), no. 2, 313-350, DOI 10.1353/ajm.0.0046. MR2503985 (2010b:14025)

[28] Judith D. Sally, Numbers of generators of ideals in local rings, Marcel Dekker Inc., New York, 1978. MR0485852 (58 \#5654)

[29] Judith D. Sally and Wolmer V. Vasconcelos, Stable rings, J. Pure Appl. Algebra 4 (1974), 319-336. MR0409430 (53 \#13185)

[30] Friedrich Karl Schmidt, Über die Erhaltung der Kettensätze der Idealtheorie bei beliebigen endlichen Körpererweiterungen (German), Math. Z. 41 (1936), no. 1, 443-450, DOI 10.1007/BF01180433. MR 1545632

[31] Charles A. Weibel, K-theory and analytic isomorphisms, Invent. Math. 61 (1980), no. 2, 177-197, DOI 10.1007/BF01390120. MR590161 (83b:13011)

[32] O. Zariski and P. Samuel, Commutative algebra Vol. 2, Van Nostrand, Princeton, 1958. MR.0090581 (19:833e)

Department of Mathematical Sciences, New Mexico State University, Las Cruces, NeW MeXico 88003-8001 